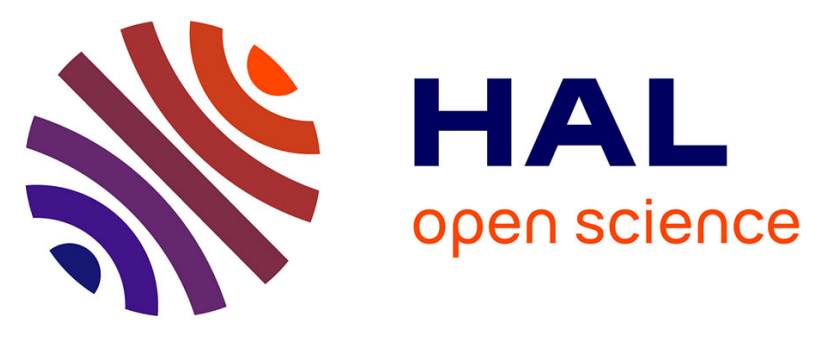

\title{
Multiscale Hill-type modeling of the mechanical muscle behavior driven by the neural drive in isometric conditions
}

Vincent Carriou, Sofiane Boudaoud, Jeremy Laforet, Adriana Mendes, Francis Canon, David Guiraud

\section{To cite this version:}

Vincent Carriou, Sofiane Boudaoud, Jeremy Laforet, Adriana Mendes, Francis Canon, et al.. Multiscale Hill-type modeling of the mechanical muscle behavior driven by the neural drive in isometric conditions. Computers in Biology and Medicine, 2019, 115, pp.\#103480. 10.1016/j.compbiomed.2019.103480 . lirmm-02307823

\section{HAL Id: lirmm-02307823 \\ https://hal-lirmm.ccsd.cnrs.fr/lirmm-02307823}

Submitted on 21 Dec 2021

HAL is a multi-disciplinary open access archive for the deposit and dissemination of scientific research documents, whether they are published or not. The documents may come from teaching and research institutions in France or abroad, or from public or private research centers.
L'archive ouverte pluridisciplinaire HAL, est destinée au dépôt et à la diffusion de documents scientifiques de niveau recherche, publiés ou non, émanant des établissements d'enseignement et de recherche français ou étrangers, des laboratoires publics ou privés.

\section{(ㄷ)(1) $\$$}

Distributed under a Creative Commons Attribution - NonCommerciall 4.0 International 


\title{
Multiscale Hill-type modeling of the mechanical muscle behavior driven by the neural drive in isometric conditions
}

\author{
Vincent Carriou $^{\mathrm{a}}$, Sofiane Boudaoud ${ }^{\mathrm{a}, *}$, Jeremy Laforet $^{\mathrm{a}}$, Adriana Mendes ${ }^{\mathrm{b}}$, Francis \\ Canon $^{\mathrm{a}}$, David Guiraud ${ }^{\mathrm{b}}$ \\ ${ }^{a}$ Sorbonne University, Universite de Technologie de Compiegne, CNRS UMR 7338 Biomechanics and \\ Bioengineering, Centre de Recherche de Royallieu - CS 60203, Compiegne, France \\ ${ }^{b}$ DEMAR Group, LIRMM, University of Montpellier 2, INRIA, CNRS, 161 rue Ada, 34095 Montpellier \\ cedex 5, France
}

\begin{abstract}
In this study, we present a new model describing the mechanical behavior of the skeletal muscle during isometric contraction. This model is based on a former Hill-inspired model detailing the electromechanical behavior of the muscle based on the Huxley formulation. However, in this new multiscale model the muscle is represented at the Motor Unit (MU) scale. The proposed model is driven by a physiological input describing the firing moments of the activated MUs. Definition of both voluntary and evoked MU recruitment schemes are described, enabling the study of both contractions in isometric conditions. During this type of contraction, there is no movement of the joints and the tendon-muscle complex remains at the same length. Moreover, some well-established macroscopic relationships such as force-length or force-velocity properties are considered. A comparison with a twitch model using the same input definition is provided with both recruitment schemes exhibiting limitations of twitch type models. Finally, the proposed model is validated with a comparison between simulated and recorded force profiles following eight electrical stimulation in isometric conditions. The simulated muscle force was generated to mimic the one recorded from the quadriceps of a patient implanted with a functional electrical stimulation neuroprosthesis. This validation demonstrates the ability of the proposed model to reproduce realistically the skeletal muscle contractions and to take into account subject-specific parameters.
\end{abstract}

Keywords: Muscle multiscale mechanical model, Isometric contraction, Motor unit,

\footnotetext{
${ }^{*}$ Corresponding author

Email address: sofiane.boudaoud@utc.fr (David Guiraud)
} 


\section{Introduction}

Ever since the mid 60s, researchers have attempted to better understand the mechanisms behind human body motion. They found that the movement is the result of several interactions between complex mechanisms in the body [1]. At the macroscopic scale, the muscle contraction is in charge of the movement genesis through the joints. In fact, during contraction, the muscle length shortens and thus it stretches the connected tendons resulting in movement of bones around a specific joint.

Several studies have been focused on the understanding of the activation $[2,3,4]$ and the mechanical phenomena of the muscle contraction $[5,6]$. Due to the complex feasibility of studying human muscles in vivo, the alternative would be to use realistic mathematical models. These models allow us a better understanding of the motor control and the different mechanisms of muscle contraction. Moreover, by controlling all the parameters of the model, users can precisely investigate the impacts of these parameters on the outputs and can, thus, be able to simulate physiological or pathological conditions.

Different approches of modeling of muscle contraction have been proposed and used in the litterature $[5,6,7,8,9,10,11]$. These models can be divided into different types based on the scale and the approach considered. First we have the phenomenological macroscopic models that are governed by Hill-Maxwell or Hill-Voigt models, inherited from the classic Hill model [5], which is generally credited as the first model describing the mechanical behavior of a muscle contraction. The Hill model describes the muscle as an active contractile component with series and parallel passive elastic components. Furthermore, the author considered the muscle as a visco-elastic material and thus, described the well-known force/length relationship of the muscle. However, this systemic macroscopic model is often described as a "black-box" driven by fitted parameters such as the muscle activation to fit the measured force. Some improved versions integrated the pennation angle of the muscle fibers with the tendon [7] and macroscopic features. Secondly, in addition to these rheological models, some biochemical microscopic models were developed. The most widely used is the model proposed by Huxley [6] which considered the muscle at the sarcomere scale. Huxley and 
al. proposed an interpretation of the cross-bridges dynamic through mathematical equations and attempted to determine the muscle force from the rates of attachment and detachment of these cross-bridges. From these two classes of muscle models, Zahalak proposed a model that bridges together the microscopic and macroscopic models [8]. From the cross-bridges model, the author computed the muscle force through mathematical approximation with the distribution-moment technique $[8,10]$. Finally, we have to mention the twitch type model introduced by Coggshall and al. [12], where the authors only described the muscle force as the sum of mechanical activities resulting from the convolution operation between the firing times of the active Motor Units (MUs) and a function defining the MU twitch. This type of model does not consider the induced muscle deformation or the underlying processes arising between the muscle activation and the force generation.

All these models provided some information about the muscle contraction and brought crucial knowledge about the muscle force generation. However, these models, in the exception of the twitch type ones, suffers from inaccuracies mainly concerning the muscle activation. Hill-type models use an identified parameter input enclosed in $[0 ; 1]$ interval representing the activation percentage of the muscle. This parameter has no physiological meaning and is hard to experimentally estimate. Other models attempted to give a sense to this activation parameter through the Functional Electrical Stimulation (FES) [10, 4, 13] where the parameter represented the percentage of muscle fiber recruited during stimulation. Yet, the recruitment pattern induced by FES does not follow voluntary contraction [13]. It reverses the natural sequence since larger MUs are recruited the first and smaller the last. Also, the FES synchronizes the firing times of all recruited MUs whereas the voluntary recruitment pattern is asynchronous. Riener and al. [13] attempted to keep the muscle representation at the MU scale. Riener and al. tried to describe the muscle force from the summation of all active MUs. They described the depolarization of the T-Tubule membrane dynamics induced by the neural activation as well as the calcium release from the sarcoplasmic reticulum in order to compute the MUs twitch. This model was the first twitch type model describing physiological processes. Recently, another type of model combining the microscopic sarcomere model of Huxley [6] with the distribution moment theory of Zahalak [8] was proposed [10]. El Makssoud and al. defined a macroscopic muscle model [10] based on the sarcomere 
model formalism and avoided the costly computation time at the sarcomere scale. From the description of the muscle with one contractile element, only one type of fiber can constitute the muscle model. Moreover, the authors also proposed a visco-elastic model describing the muscle-tendon contraction during isometric contraction. This model was validated on both animal and human subjects [10, 4].

The major increment of the proposed model is to drive the mechanical muscle response with the voluntary MU spatiotemporal recruitment scheme using specific Hill inspired elements at the MU scale including fiber population. In [10], the proposed mechanical model represented the whole muscle response with an unspecific fiber stack. Furthermore, the model was driven by a single input (FES input). This mechanical modeling at the MU scale combining Hill-type representations and microscopic sarcomere model does not exist, to our knowledge, in the literature. Moreover, using this activation definition was never implemented in Hill-type models where the sEMG signal envelope was often used. This classical activation definition suffers from a lack of representativeness of the underlying neural intent during the contraction. In our proposed model, the muscle is represented at the MU scale where the MU contractile elements are repeated in parallel to constitute the muscle. Firstly, we detail the different models used for the simulation (see sections 2.1, 2.2 and 2.3). Then, some results showing the efficiency of our model are presented and discussed (see section 3). Finally, we conclude by emphasizing the main contribution of this study and the possible perspectives.

\section{Materials and methods}

As introduced, the main purpose of this study is to propose a mechanical multiscale modeling of the skeletal muscle driven by voluntary neural control. This model would be a significant improvement of the model presented above $[5,6,8,13,10]$. The underlying purpose is also to provide a bio-reliable model which is based on physiological parameters to get significant results that can help in a better description of the muscle contraction for

clinical purposes. Each MU will be modeled by one specific contractile element placed in parallel to model the whole muscle. Thus, the proposed muscle model is divided into two parts as presented in Fig. 1: 
- the first part represents the activation model of the muscle and gives a physiologic meaning of the input of the mechanical model;

- the second part is the muscle mechanical model at the MU scale based on the distributed moment technique inspired by [10].

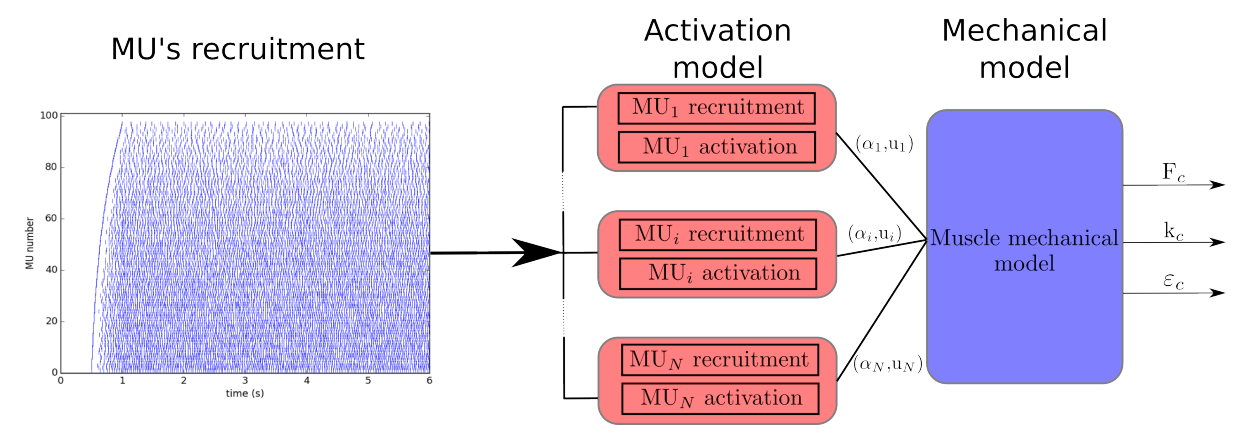

Figure 1: Model block diagram. On the left, there is the input of the model corresponding to the recruitment pattern describing the discharge instant of the recruited MUs. On the right, the mechanical model scheme, determining the activation $\left(\alpha_{i}\right)$ and calcium release $\left(u_{i}\right)$ of each MUs then, processes at the muscle scale in order to determine the muscle force $\left(F_{c}\right)$, the muscle stiffness $\left(k_{c}\right)$ and its deformation $\left(\varepsilon_{c}\right)$.

The first challenge is to provide an activation model based on the firing instants of the active MUs.

\subsection{Motor Unit recruitment model}

\subsubsection{Voluntary recruitment}

During contraction, MUs are recruited independently of others and in agreement with the size principle [14]. Each MU is activated at a specific threshold depicted in the Fuglevand model [15]. However, Kukulka and Clamann [16] have provided evidence that the distribution of the recruitment threshold for larger proximal muscles, such as the Biceps Brachii, is less skewed compared with the exponential distribution of other muscles. Therefore, we decided to use the following equation from which is an extension of the Fuglevand model [17]:

$$
R T E_{i}=\left(\frac{a . i}{N}\right) e^{i \frac{\ln \left(\frac{R R}{a}\right)}{N}}
$$

where $R T E_{i}$ is the recruitment threshold for the $i^{\text {th }} \mathrm{MU}, N$ is the total number of $\mathrm{MU}$ in the muscle and $a=40$ is a constant value describing the slope of the recruitment threshold 
function [17]. It is also tuned by the recruitment range $(R R=88 \%[16])$ which describes the percentage of the Maximal Voluntary Contraction (MVC) when all MUs are recruited in the muscle.

Each MU recruitment is regulated by a motoneuron firing rate $\left(F r_{i}\right)$ which increases linearly or non-linearly with force level, from its minimal firing rate $\left(F r_{\min }\right)$ to its peak firing rate $\left(P F R_{i}\right)$. In this model, for each $\mathrm{MU}, P F R_{i}$ is taken inversely proportional to the recruitment threshold for the linear increase of firing rate.

$$
\begin{gathered}
F r_{i}(t)=g_{i}\left(E(t)-R T E_{i}\right)+F r_{\text {min }} \text {, if } E(t) \geq R T E_{i} \\
g_{i}=\frac{P F R_{i}-F r_{\text {min }}}{100_{\% M V C}-R T E_{i}} \\
P F R_{i}=P F R_{1}-P F R D \frac{R T E_{i}}{R T E_{N}}
\end{gathered}
$$

Where $E(t)$ is the excitatory drive provided by the Central Nervous System. The firing frequencies $F r_{\min }$ and $P F R_{1}$ are fixed to 8 and $35 \mathrm{~Hz}$, respectively [17]. $P F R D$ is the peak firing rate difference and is fixed to $15 \mathrm{~Hz}$ in this study. It accounts for the simulation of the "onion skin" phenomenon observed in several experimental studies [18].

Moreover, the model also considers the MU type since it is well-known that most of the skeletal muscles are composed of four types of fiber and thus, of MU:

- Slow or type I MUs (SMUs) that provide a low force with a slow contraction speed and has a high resistance to fatigue;

- Fast Resistant or type IIa MUs (FRMUs) that provide an intermediate force, with fast contraction speed and are resistant to fatigue;

- Fast Intermediate or type IIx MUs (FIMUs) that provide an intermediate force (more than FRMU), with fast contraction speed and are less resistant to fatigue than FRMU;

- Fast Fatigable or type IIb MUs (FFMUs) that provide high force, with very fast contraction speed but are quickly fatigued. 


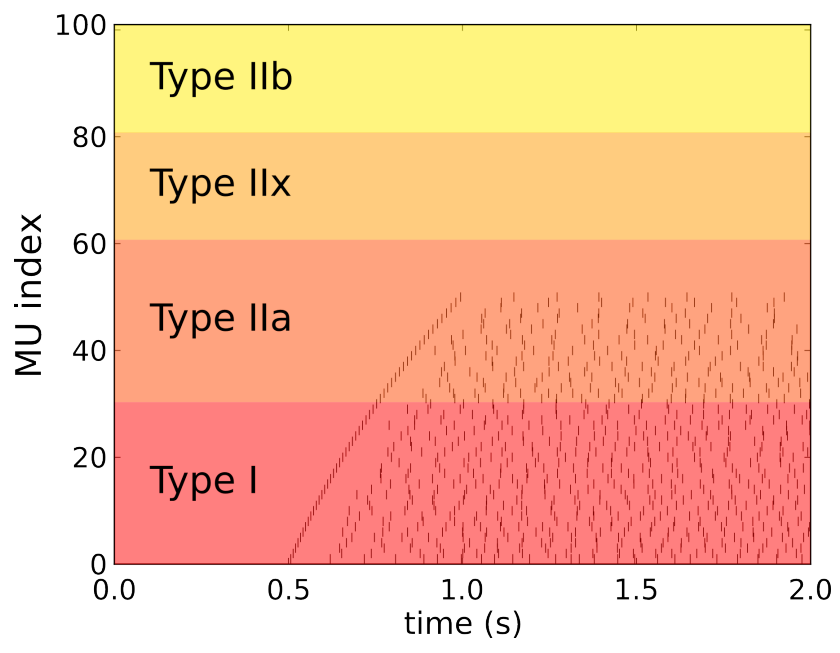

Figure 2: Example of simulated voluntary MU recruitment starting at $0.5 \mathrm{~s}$ from $0 \%$ to $30 \%$ (at $1.0 \mathrm{~s}$ ) of the Maximum Voluntary Contraction (MVC) for a muscle composed of 100 MUs with the following MUs distribution type: $33 \%$ type I, 33\% type IIa, 17\% type IIx and 17\% type IIb. Each point represents a firing moment of the MU.

\subsubsection{Evoked recruitment model}

In addition to the voluntary recruitment, an evoked recruitment of the MUs simulating a Neural Electrical Stimulation (NES) is also developed in this model. This type of recruitment is often used in mechanical muscle study to exactly quantify the muscle activation parameter $[13,10]$. During a NES, the MUs are recruited synchronously with respect to the diameter size of their motoneuron and the area of electrical spread. Larger motoneurons, representing larger MU radius, are hired first in descending order [19]. The number of recruited MUs mostly depends on the current intensity and the pulse width of the NES [2]. The number of contracting MUs is bigger with a higher intensity and a longer pulse width. For this purpose, we have used the same equation as in [4] that describes the recruitment, corresponding to the recruited percentage of the muscle, (noted $\alpha$ ) according to the pulse width.

$$
\alpha(p w)=\frac{c_{1}}{1+e^{c_{2}\left(c_{3}-\frac{p w}{p w w_{\text {max }}}\right)}}
$$

with $p w_{\max }$ as the maximal pulse width depending on the stimulator device. $c_{1}, c_{2}$ and $c_{3}$ are parameters that represent the plateau level, maximum slope and inflection point, respectively. These parameters can be computed using an identification algorithm as in [4]. 
This description ensures a monotonic increase of eq (3) assuring function $\alpha$ increase according to the input pulse width $p w$. Moreover, this equation can be adapted considering the current intensity. Function $\alpha$ is no longer a function of the pulse width but of the current intensity giving:

$$
\alpha(i)=\frac{c_{1}}{1+e^{c_{2}\left(c_{3}-\frac{i}{i m a x}\right)}}
$$

where $i_{\max }$ is the maximal current intensity depending on the stimulator.

Then, from the recruitment $\alpha$ representing the percentage of recruited fibers in the muscle, we generated the corresponding evoked recruitment pattern (see Fig. 3). Since we know the total number of fibers in the muscle and the number of fibers per MU from the anatomy generated by [20], we can create the corresponding recruitment pattern of the NES.

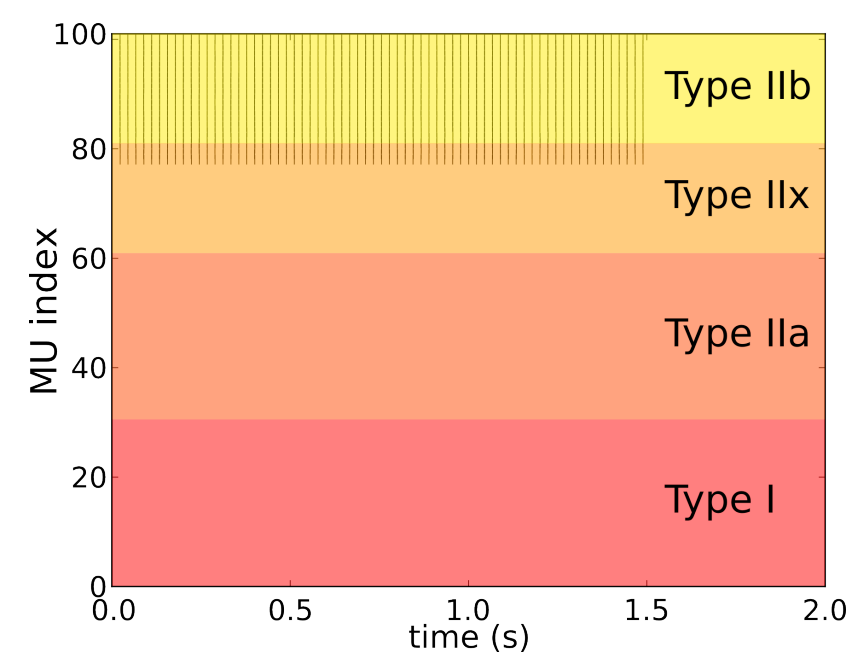

Figure 3: Example of evoked recruitment where $32.4 \%$ of the muscle fibers are recruited for the same muscle defined in Fig. 2.

\subsection{Activation model}

Previously, the mechanical model input was the percentage of recruited muscle fibers determined with FES [10]. This input description cannot be applied for voluntary contractions and thus, restricts the use of this model type. The purpose of this study is to simulate accurate contractile behaviors of the muscle governed by a MU voluntary or evoked recruitment pattern. We based our model development on physiological processes arising during the contraction [13]. 
Each MU type will have its own set of parameters for both activation and mechanical characteristics corresponding to their different properties. As in $[6,13,10]$, we considered that the fiber contraction is dependent on the calcium ion $\left(\mathrm{Ca}^{2+}\right)$ concentration presents in the cytoplasm. From this hypothesis, MUs can be in one of these three states during the muscle contraction: in contraction, in relaxation or relaxed. When a MU is recruited, it will contract during a determined time, according to the $\mathrm{Ca}^{2+}$ release. If it does not receive a new impulse during the contraction time, the MU starts to be in relaxation according to the $\mathrm{Ca}^{2+}$ uptake time. Finally, if it still does not receive a new impulse it goes in relaxed state when its $\mathrm{Ca}^{2+}$ concentration reaches the concentration at rest. Thus, these states will be defined according to the intracellular $\mathrm{Ca}^{2+}$ dynamic.

\subsubsection{Calcium dynamics}

When a neural Action Potential (AP) reaches the fiber neuromuscular junction, an action potential traveling along the sarcolemma is created. During the AP propagation, the sarcoplasmic reticulum (SR) releases $\mathrm{Ca}^{2+}$ into the myoplasm [21]. When this concentration exceeds a specific threshold, the fibers within the MU begin to contract. The contraction remains until this concentration decreases below the threshold. Detailed models of the calcium concentration dynamics are presented in $[22,23,13,24]$. However, we decided to use the definition of the $\mathrm{SR} \mathrm{Ca}^{2+}$ release in response to an AP from [24] adapted to the MU type.

$$
u_{i}(t)=\left\{\begin{array}{cc}
U_{r, i} & \text { if } t<\tau_{D} \\
R_{i} \cdot\left(1-e^{-\left(\frac{t-\tau_{D}}{\tau_{1, i}}\right)}\right)^{5} \cdot e^{-\left(\frac{t-\tau_{D}}{\tau_{2, i}}\right)} & \text { if } t \geq \tau_{D}
\end{array}\right.
$$

Where $i$ represents the $i^{\text {th }} \mathrm{MU}, U_{r, i}$ is the $\mathrm{Ca}^{2+}$ concentration at rest according to the MU type of $\mathrm{MU} i, \tau_{D}$ is the time delay response to the $\mathrm{Ca}^{2+}$ release which is the same for all type of $\mathrm{MU}, R_{i}$ is an amplitude parameter to be in agreement with the calcium amplitude according to the type of the $\mathrm{MU} i$ and $\tau_{1, i}$ and $\tau_{2, i}$ are specific parameters according to the MU type and representing the rise and the descent of the $\mathrm{Ca}^{2+}$ dynamics, respectively. This calcium dynamic is depicted in Fig. 4.

The contraction-relaxation cycle is formed to follow two phases: every time an AP reaches a MU, a contraction begins with a time delay of $\tau_{D}$ for taking into account the propagation time of the AP and the average time delay due to the calcium dynamics. After this delay, a 

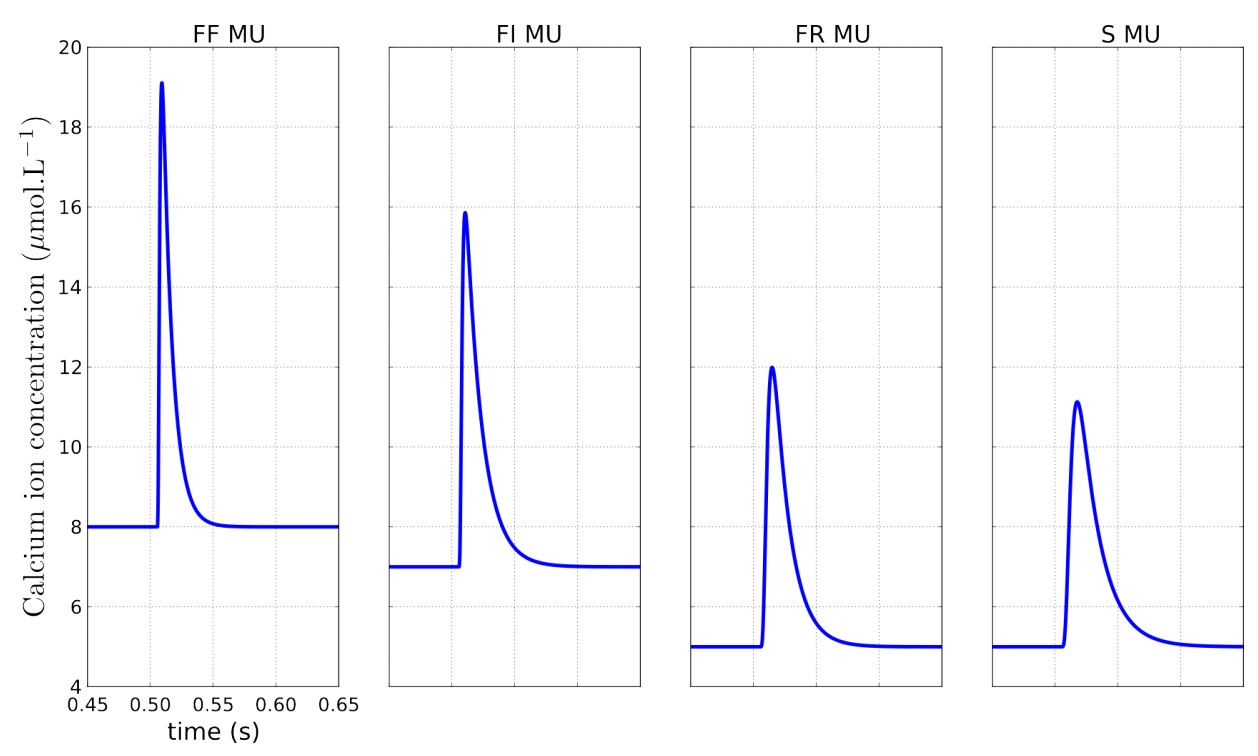

Figure 4: Calcium dynamic of MU according to its type after a neural activation at $0.5 \mathrm{~s}$.

contraction phase takes place and $\mathrm{Ca}^{2+}$ concentration ups above the calcium threshold $\left(U_{c, i}\right)$ during a few moments according to the MU type. After this, if no other AP has been received in the meantime, an active relaxation follows until the rest $\mathrm{Ca}^{2+}$ concentration $U_{r, i}$ is reached. The calcium release is fast enough in skeletal muscle to be considered quasi-instantaneous (see Fig. 4). These dynamics will be determined using the convolution operator between the firing times of the corresponding $\mathrm{MU}$ and the calcium function. In this model, the calcium kinetics is the same for all MUs with the same type. In Table 1 is presented the values of the parameters defining the calcium dynamics according to the MU type. Parameters in this table have been determined based on results from the SR release [25] to represent physiological behavior according to MU type. Compared to [24], the time response $\left(\tau_{D}\right)$ is higher. However, we fixed this value in order to correspond to the electromechanical delay of the twitch model [26] since the mechanical response starts instantaneously when the calcium dynamic goes above the contraction threshold. 
Table 1: Parameters defining the calcium dynamics according to MU type

\begin{tabular}{|c|c|}
\hline Parameter & Value (unit) \\
\hline Time delay response $\left(\tau_{D}\right)$ & $5(\mathrm{~ms})[26]$ \\
\hline SMU $\mathrm{Ca}^{2+}$ concentration at rest $\left(U_{r, S}\right)$ & $5\left(\mu \mathrm{mol} . \mathrm{L}^{-1}\right)$ \\
\hline FRMU $\mathrm{Ca}^{2+}$ concentration at rest $\left(U_{r, F R}\right)$ & $5\left(\mu \mathrm{mol} . \mathrm{L}^{-1}\right)$ \\
\hline FIMU $\mathrm{Ca}^{2+}$ concentration at rest $\left(U_{r, F I}\right)$ & $7\left(\mu \mathrm{mol} . \mathrm{L}^{-1}\right)$ \\
\hline FFMU $\mathrm{Ca}^{2+}$ concentration at rest $\left(U_{r, F F}\right)$ & $8\left(\mu \mathrm{mol} . \mathrm{L}^{-1}\right)$ \\
\hline SMU rise slope $\left(\tau_{1, S}\right)$ & $4(\mathrm{~ms})$ \\
\hline SMU descent slope $\left(\tau_{2, S}\right)$ & $20(\mathrm{~ms})$ \\
\hline SMU amplitude calcium dynamics $\left(R_{S}\right)$ & 16 \\
\hline FRMU rise slope $\left(\tau_{1, F R}\right)$ & $3(\mathrm{~ms})$ \\
\hline FRMU descent slope $\left(\tau_{2, F R}\right)$ & $17(\mathrm{~ms})$ \\
\hline FRMU amplitude calcium dynamics $\left(R_{S}\right)$ & 16 \\
\hline FIMU rise slope $\left(\tau_{1, F I}\right)$ & $1.3(\mathrm{~ms})$ \\
\hline FIMU descent slope $\left(\tau_{2, F I}\right)$ & $13(\mathrm{~ms})$ \\
\hline FIMU amplitude calcium dynamics $\left(R_{S}\right)$ & 14.5 \\
\hline FFMU rise slope $\left(\tau_{1, F F}\right)$ & 1 (ms) \\
\hline FFMU descent slope $\left(\tau_{2, F F}\right)$ & $8(\mathrm{~ms})$ \\
\hline FFMU amplitude calcium dynamics $\left(R_{S}\right)$ & 20.0 \\
\hline
\end{tabular}

\subsection{2. $M U$ activation model}

During muscle contraction, the MU can be in one of these three states: activated $\left(\alpha_{i}(t)\right)$, in relaxation $\left(\beta_{i}(t)\right)$ and relaxed $\left(\gamma_{i}(t)\right)$. This activation can be determined from the calcium dynamics of the MU defined above (see section 2.2.1). From this definition, we proposed these activation equations describing $\mathrm{MU}$ state in time:

$$
\alpha_{i}(t)=\left\{\begin{array}{ccc}
1 & \text { if } & u_{i}(t) \geq U_{c, i} \\
0 & \text { otherwise }
\end{array}\right.
$$




$$
\begin{gathered}
\beta_{i}(t)=\left\{\begin{array}{ccc}
1 & \text { if } & U_{r, i}<u_{i}(t)<U_{c, i} \\
0 & \text { otherwise }
\end{array}\right. \\
\gamma_{i}(t)=\left\{\begin{array}{lc}
1 & \text { if } \\
0 & \text { otherwise }
\end{array} u_{i}(t)=U_{r, i}\right.
\end{gathered}
$$

By weighting the MU activation parameter with its number of fibers divided by the total number of fibers within the muscle, we can obtain the muscle activation state related to the percentage of the muscle that is contracted, in relaxation and relaxed during time as we can see in Fig. 5. Table 2 presents the parameter values used in this model.

Table 2: Fixed parameters

\begin{tabular}{|c|c|}
\hline Concentration activated threshold & Value (unit) \\
\hline SMU Ca ${ }^{2}+\left(U_{c, S}\right)$ & $10\left(\mu \mathrm{mol} . \mathrm{L}^{-1}\right)$ \\
\hline FRMU Ca ${ }^{2}+\left(U_{c, F R}\right)$ & $11\left(\mu \mathrm{mol}_{\mathrm{L}}{ }^{-1}\right)$ \\
\hline FIMU Ca ${ }^{2}+\left(U_{c, F I}\right)$ & $14\left(\mu \mathrm{mol} . \mathrm{L}^{-1}\right)$ \\
\hline FFMU Ca ${ }^{2}+\left(U_{c, F F}\right)$ & $15\left(\mu \mathrm{mol}_{\mathrm{L}}{ }^{-1}\right)$ \\
\hline
\end{tabular}
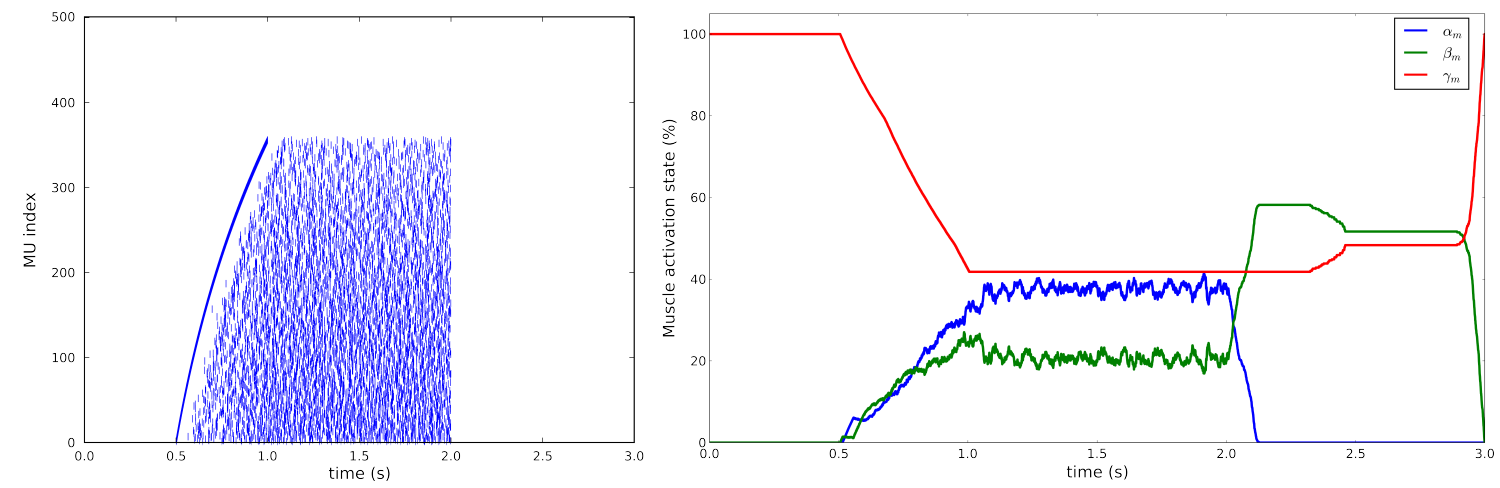

Figure 5: Muscle activation states $\alpha, \beta$ and $\gamma$ (Right) according to a voluntary contraction at 50\% MVC (Left). No relaxation model is considered in this modeling thus, once the contraction task is over, all the MUs stop to be recruited instantaneously. 


\subsection{Mechanical model of the muscle during isometric contraction}

Previously, the muscle mechanical model was developed only at the muscle scale. In the proposed model, we attempt to provide a mechanical model at the MU scale. Unlike models in $[5,7,8,10]$, the muscle is modeled by $N$ contractile elements with the same length $L_{c}$ placed in parallel. Each contractile element will describe the mechanical dynamics of one MU.

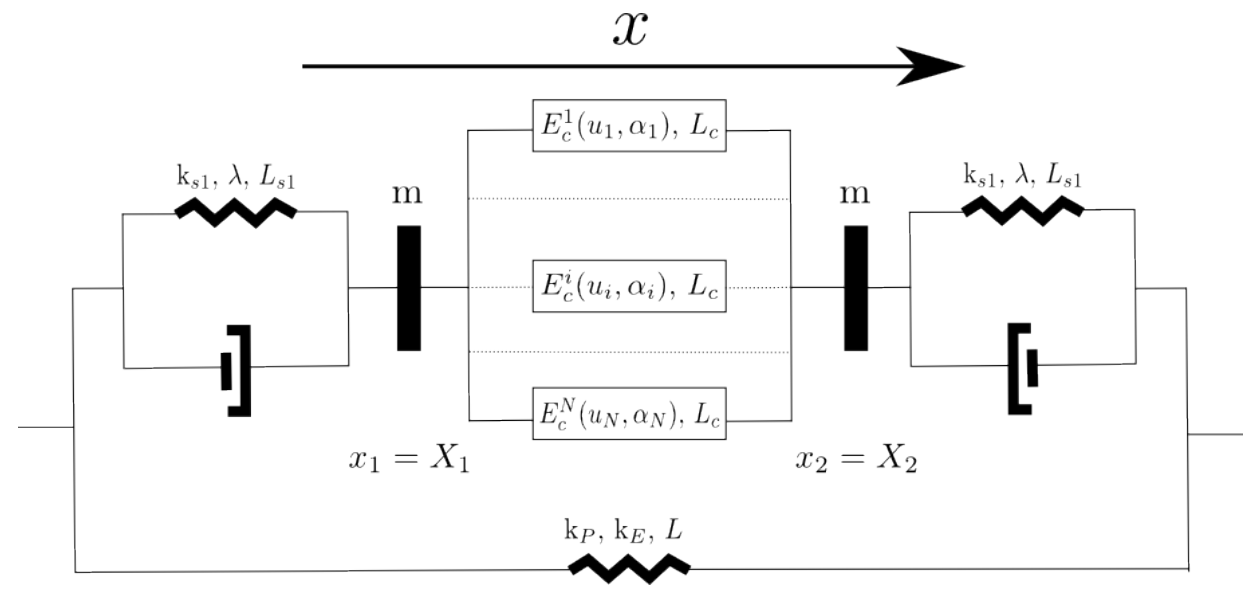

Figure 6: Mechanical rheological model of the muscle, including masses and dampers derived from [10] with $N$ parallel contractile elements representing the MU. $X_{1}$ and $X_{2}$ are the positions of the muscle-tendon junction when the muscle is at rest.

This model presented in Fig. 6 is composed of macroscopic passive elements interacting with $N$ contractile elements $E_{c}^{i}$ that contract independently and with its local activation described in section 2.2.2. We assume the left and right tendons having the same properties. To stay close to the structure proposed by Hill, the springs $k_{s 1}$ are assumed to be linear. $\mathrm{L}_{s 10}$ is the length of the springs at rest condition when no force is generated and $\mathrm{L}_{s 1}$ is the spring length at any time. $\mathrm{L}$ is the length of the parallel spring and $\mathrm{L}_{c 0}$ is defined as the length at which the muscle produces the maximum isometric force and $m$ is the muscle mass. From this model we have:

$$
\left\{\begin{array}{l}
m \ddot{x_{1}}=F_{c}+k_{s 1}\left(X_{1}-x_{1}\right)+\lambda\left(X_{1}-x_{1}\right) \\
m \ddot{x_{2}}=-F_{c}+k_{s 1}\left(X_{2}-x_{2}\right)-\lambda\left(X_{2}-x_{2}\right)
\end{array}\right.
$$

Subtracting these two dynamics leads to: 


$$
m\left(\ddot{x_{2}}-\ddot{x_{1}}\right)=-2 F_{c}+k_{s 1}\left(X_{2}-x_{2}-X_{1}+x_{1}\right)+\lambda\left(\dot{x_{1}}-\dot{x_{2}}\right)
$$

Knowing that : $\left(x_{2}-x_{1}\right)=L_{c}$ and $\left(X_{2}-X_{1}\right)=L_{c}^{0}$, the rest length of the muscle, we obtain:

$$
m \ddot{L_{c}}=-2 F_{c}+k_{s 1}\left(L_{c}^{0}-L_{c}\right)-\lambda \dot{L_{c}}
$$

with $\varepsilon_{c}=\frac{L_{c}-L_{c 0}}{L_{c 0}} \Rightarrow L_{c}=\varepsilon_{c} L_{c 0}+L_{c 0}$.

Equation (11) becomes:

$$
\begin{gathered}
m L_{c 0} \ddot{\varepsilon}_{c}=-2 F_{c}-k_{s 1}\left(L_{c}^{0}-\varepsilon_{c} L_{c 0}-L_{c 0}\right)-\lambda \dot{\varepsilon}_{c} L_{c 0} \\
\Rightarrow \ddot{\varepsilon}_{c}=-\frac{2 F_{c}}{m L_{c 0}}-\frac{k_{s 1}}{m} \varepsilon_{c}-\frac{\lambda}{m} \dot{\varepsilon_{c}}-\frac{k_{s 1}}{m}+\frac{k_{s 1} L_{c}^{0}}{m L_{c 0}}
\end{gathered}
$$

Moreover, by definition we have: $L_{c}^{0}=\varepsilon_{c}^{0} L_{c 0}+L_{c 0}$. Where $\varepsilon_{c}$, defined above, represents the relative deformation of the muscle compared to $L_{c 0}$. Thus, equation (13) becomes:

$$
\Rightarrow \ddot{\varepsilon}_{c}=-\frac{2 F_{c}}{m L_{c 0}}-\frac{k_{s 1}}{m} \varepsilon_{c}-\frac{\lambda}{m} \dot{\varepsilon_{c}}-\frac{k_{s 1}}{m}+\frac{k_{s 1}\left(\varepsilon_{c}^{0} L_{c 0}+L_{c 0}\right)}{m L_{c 0}}
$$

Finally,

$$
\Rightarrow \ddot{\varepsilon}_{c}=-\frac{2 F_{c}}{m L_{c 0}}-\frac{k_{s 1}}{m} \varepsilon_{c}-\frac{\lambda}{m} \dot{\varepsilon}_{c}+\frac{k_{s} 1}{m} \varepsilon_{c}^{0}
$$

Then, from (10), we also have:

$$
2 F_{c}=-m L_{c 0} \ddot{\varepsilon}_{c}+k_{s 1}\left(L_{c}^{0}-L_{c}\right)-\lambda \dot{L}_{c}
$$

Knowing that in isometric contraction we have: $L_{c}+2 L_{s 1}=L=L_{c}^{0}+2 L_{s 10}$ and $L_{c 0} \varepsilon_{c}+$ $2 L_{s 10} \varepsilon_{s 1}-2 L_{s 10} \varepsilon_{s 1}^{0}=0$. With $\varepsilon_{s 1}^{0}=\frac{L_{s 1}^{0}-L_{s 10}}{L_{s 10}}$ is the relative deformation of the tendon between its length at rest $\left(L_{s 10}\right)$ and its optimal length $\left(L_{s 1}^{0}\right)$, equation $(16)$ becomes:

$$
\begin{gathered}
2 F_{c}=2 m L_{s 10} \ddot{\varepsilon}_{s 1}+2 k_{s 1}\left(L_{s 1}-L_{s 10}\right)+2 \lambda \dot{L}_{s 1} \\
\Rightarrow F_{c}=m L_{s 10} \ddot{\varepsilon}_{s 1}+k_{s 1} L_{s 10} \varepsilon_{s 1}+\lambda L_{s 10} \dot{\varepsilon}_{s 1}
\end{gathered}
$$


Moreover, the force $F_{e}$ of the visco-elastic component representing the tendon is the sum of the spring force $F_{s}$ and the damper force $F_{d}$. Thus, $F_{e}=F_{s}+F_{d}=k_{s 1} L_{s 10} \varepsilon_{s 1}+\lambda L_{s 10} \dot{\varepsilon}_{s 1}$. When the ratio of $F_{c}$ and $F_{e}$ is considered using Laplace transform, we have:

$$
\frac{\mathcal{L}\left[F_{c}\right]}{\mathcal{L}\left[F_{e}\right]}=\frac{m s^{2} \Upsilon(s)+\lambda s \Upsilon(s)+k_{s 1} \Upsilon(s)}{\lambda s \Upsilon(s)+k_{s 1} \Upsilon(s)}
$$

With $\Upsilon(s)$ is the Laplace transform of $L_{s 10} \varepsilon_{s 1}(t)$. Thus, we can write:

$$
\lambda s \mathcal{L}\left[F_{c}\right]+k_{s 1} \mathcal{L}\left[F_{c}\right]=m s^{2} \mathcal{L}\left[F_{e}\right]+\lambda s \mathcal{L}\left[F_{e}\right]+k_{s 1} \mathcal{L}\left[F_{e}\right]
$$

Giving, after inverse Laplace transform use, the following differential equation:

$$
\begin{gathered}
m \ddot{F}_{e}+\lambda \dot{F}_{e}+k_{s 1} F_{e}=\lambda \dot{F}_{c}+k_{s 1} F_{c} \\
\Rightarrow \ddot{F}_{e}=-\frac{\lambda}{m} \dot{F}_{e}-\frac{k_{s 1}}{m} F_{e}+\frac{\lambda}{m} \dot{F}_{c}+\frac{k_{s 1}}{m} F_{c}
\end{gathered}
$$

Finally, we have this system at the muscle scale:

$$
\left\{\begin{array}{l}
\ddot{\varepsilon}_{c}=\frac{k_{s 1}}{m} \varepsilon_{c}^{0}-\frac{2 F_{c}}{m L_{c 0}}-\frac{k_{s 1}}{m} \varepsilon_{c}-\frac{\lambda}{m} \dot{\varepsilon}_{c} \\
\ddot{F}_{e}=-\frac{\lambda}{m} \dot{F}_{e}-\frac{k_{s 1}}{m} F_{e}+\frac{\lambda}{m} \dot{F}_{c}+\frac{k_{s 1}}{m} F_{c}
\end{array}\right.
$$

An equation system is also needed for each contractile element representing a MU. According to [10], we have, for each contractile element, the dynamic of its stiffness $k_{c, i}$ and its force $F_{c, i}$ :

$$
\left\{\begin{aligned}
\dot{k}_{c, i}(t)= & -\left(u_{i}(t)+a\left|\dot{\varepsilon}_{c}(t)\right|\right) k_{c, i}(t)+\alpha_{i}(t) k_{\max , i}(t) \Pi_{c, i}(t) U_{c, i} \\
\dot{F}_{c, i}(t)= & -\left(u_{i}(t)+a\left|\dot{\varepsilon}_{c}(t)\right|\right) F_{c, i}(t)+\alpha_{i}(t) F_{\max , i}(t) \Pi_{c, i}(t) U_{c, i}+ \\
& \omega\left(F_{c, i}(t)\right) k_{c}(t) L_{c 0} \dot{\varepsilon}_{c}(t)
\end{aligned}\right.
$$

With, $F_{c}=\sum_{i=0}^{N} F_{c, i}$ and $k_{c}=\sum_{i=0}^{N} k_{c, i}$ the muscle force and stiffness, respectively. $u_{i}$ is the calcium dynamic described in section 2.2.1, $a$ is a parameter describing the velocity contribution between the actin and myosin filaments, $\alpha_{i}$ is the activation parameter described in section 2.2.2, $k_{\max , i}$ and $F_{\max , i}$ are the maximum stiffness and force produced by the 
$i^{\text {th }} \mathrm{MU} . U_{c, i}$ is the calcium concentration threshold of the $\mathrm{i}^{\text {th }} \mathrm{MU}$ determining when the contraction occurs. Moreover, $\Pi_{c, i}$ is a function following the normalized calcium dynamic according to the MU type (see eq 5). Assuming that all the fibers have the same mechanical contribution, $k_{\max , i}(t)$ and $F_{\max , i}(t)$ are determined as a percentage of the maximal stiffness $\left(k_{\max }\right)$ and force $\left(F_{\max }\right)$ produced by the muscle according to the number of innervated fiber in the MU. Moreover, $k_{\max , i}(t)$ and $F_{\max , i}(t)$ are function of the deformation $\varepsilon_{c}$ described as follow:

$$
\left\{\begin{array}{l}
F_{\max , i}(t)=F_{\max , i} f_{l}\left(\varepsilon_{c}\right) \\
k_{\max , i}(t)=k_{\max , i} f_{l}\left(\varepsilon_{c}\right)
\end{array}\right.
$$

with

$$
f_{l}\left(\varepsilon_{c}\right)=e^{\frac{-\varepsilon_{c}^{2}}{b}}
$$

where $b$ is a scale parameter. Finally, $\omega\left(F_{c}\right)$ (in equation $\left.(23)\right)$ is defined as follows:

$$
\omega\left(F_{c}\right)=\left\{\begin{array}{cc}
1 & \text { if } F_{c}>10^{-4} \\
0 & \text { otherwise }
\end{array}\right.
$$

This window function is used to prevent the passive force generated by the non recruited MUs that should not produce active force. Details of the calculus to obtain the system (23) can be obtained from [10].

\section{Results}

In all the results present below, some parameters were set. These parameters are presented in tables 1 and 2 .

At first, we propose to study the fusion frequency using the proposed model on the same muscle with only different MU type distribution (see 3.1.1). Then, thanks to the MU scale modeling, we propose to study these frequencies at the MU scale regarding the MU type (see 3.1.2). Afterward, we suggest doing the comparison of the fusion frequency between the proposed model and the twitch model (see 3.1.3). This comparison is then extended to voluntary isometric contractions (see 3.2). Finally, a validation of the model is proposed with experimental data extracted from the study in [4] (see 3.3). 


\subsection{Fusion frequency study}

\subsubsection{Muscle scale}

In this subsection, we will present some results concerning the fusion frequency needed to simulate the twitch fusion according to the MU type. For this purpose, we simulated two muscles composed of $500 \mathrm{MUs}$ using the following distributions of MU type: 33\% SMU, 17\% FRMU, 17\% FIMU and 33\% FFMU for the first muscle $\left(\mathrm{M}_{1}\right)$ and 33\% SMU, 33\% FRMU, $17 \%$ FIMU and 17\% FFMU for the second muscle $\left(\mathrm{M}_{2}\right)$. This difference between these two muscles is the percentage of MUs considering their force generation. In $\mathrm{M}_{1}$ there are 250 MUs that are considered low (S and FR MUs) regarding their force production whereas 330 are defined in $\mathrm{M}_{2}$. These two configurations physiologically mimic fast $\left(\mathrm{M}_{2}\right)$ and slow $\left(\mathrm{M}_{1}\right)$ muscle respectively. Concerning the MU recruitment, it was defined using the description in section 2.1.2 and equation (3). Parameters of this equation were set in order to recruit all the MUs at each stimulation (see Table 3).

Different frequencies of stimulation were used in this study, according to [26, 27], the frequency fusion of MU mechanical response to a stimulus is correlated to its type. Thus, we proposed to simulate for the same muscle and parameters of the model, 4 different fusion frequencies, 5, 20, 50 and $100 \mathrm{~Hz}$. The simulation's parameters set is presented in Table 3.

Using this set of parameters will recruit $100 \%$ of the MUs in the muscle. The fusion frequency comparison can be made on the different mechanical behavior of the muscle (force, stiffness or deformation). In this section, we decided to compare the force generation according to the stimulation frequency using the recruitment defined above in Fig. 3.

In Fig. 7, we can observe an increase in the generated force according to the stimulation frequency increase. We can also observe the force fusion increasing with the frequency from the amplitude reduction between impulses. Thus, the optimal fusion frequency for these muscles is around $100 \mathrm{~Hz}$. 
Table 3: Parameters used for the fusion frequency

\begin{tabular}{|c|c|}
\hline Parameter & Value (unit) \\
\hline Maximal intensity $\left(i_{\max }\right)$ & $3.15(\mathrm{~mA})[4]$ \\
\hline Intensity $(i)$ & $3.15(\mathrm{~mA})$ \\
\hline Ellicited recruitment parameters $\left(c_{1}, c_{2}, c_{3}\right)$ & $1.15,8.12,0.75[4]$ \\
\hline Optimal muscle length $\left(L_{c 0}\right)$ & $9.18(\mathrm{~cm})$ \\
\hline Rest muscle length $\left(L_{c}^{0}\right)$ & $10.0(\mathrm{~cm})$ \\
\hline Velocity contribution $(a)$ & $1.0[4]$ \\
\hline Force-length parameter $(b)$ & $0.54[4]$ \\
\hline Tendon viscosity $(\lambda)$ & $19.0\left(\mathrm{~kg} . \mathrm{s}^{-1}\right)[10]$ \\
\hline Muscle weight $(m)$ & $0.5(\mathrm{~kg})$ \\
\hline Maximal force $\left(F_{\max }\right)$ & $300(\mathrm{~N})$ \\
\hline Maximal stiffness $\left(k_{\max }\right)$ & $100 \mathrm{~N} . \mathrm{mm}^{-1}$ \\
\hline Tendon stiffness $\left(k_{s 1}\right)$ & $120 \mathrm{~N} . \mathrm{mm}^{-1}$ \\
\hline
\end{tabular}
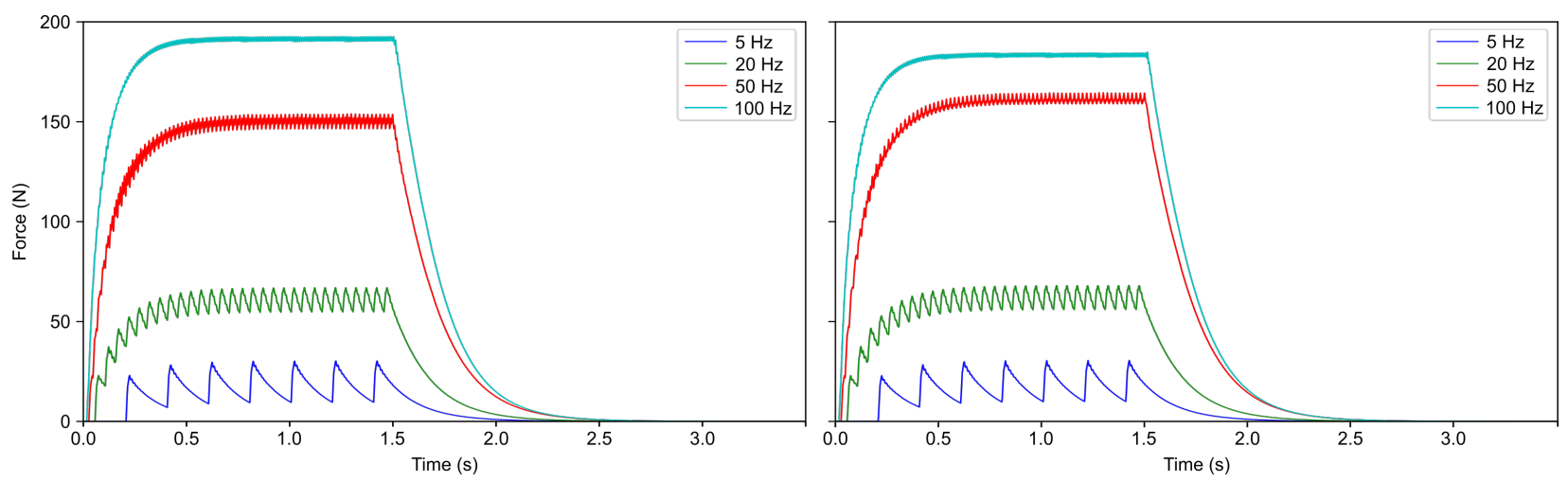

Figure 7: (L) Fused and unfused tetani according to the stimulation frequency for muscle $\mathrm{M}_{1}$. The first stimulation pulse appends according to the stimulation frequency, i.e., for $5 \mathrm{~Hz}$ the first impulse arises at $0.2 \mathrm{~s}$. (R) Fused and unfused tetani according to the stimulation frequency for muscle $\mathrm{M}_{2}$.

Moreover, we observe lower generated force with $M_{2}$ muscle than $M_{1}$ muscle at $100 \mathrm{~Hz}$. In fact, $\mathrm{M}_{2}$ muscle has more slow MUs that generate less force than $\mathrm{M}_{1}$ muscle that has more fast MUs. This difference could be explained by the MU type distribution, knowing 
that SMUs generate less force than FFMUs. Yet, at $50 \mathrm{~Hz}$ the $\mathrm{M}_{2}$ generates more force than $\mathrm{M}_{1}$. Thus, we can suppose that the optimal fusion frequency for $\mathrm{M}_{2}$ muscle is around $50 \mathrm{~Hz}$. Finally, at 5 and $20 \mathrm{~Hz}$, only partial fusion arises and gives almost the same results for both simulated muscles. These differences are exhibited in Fig. 8.

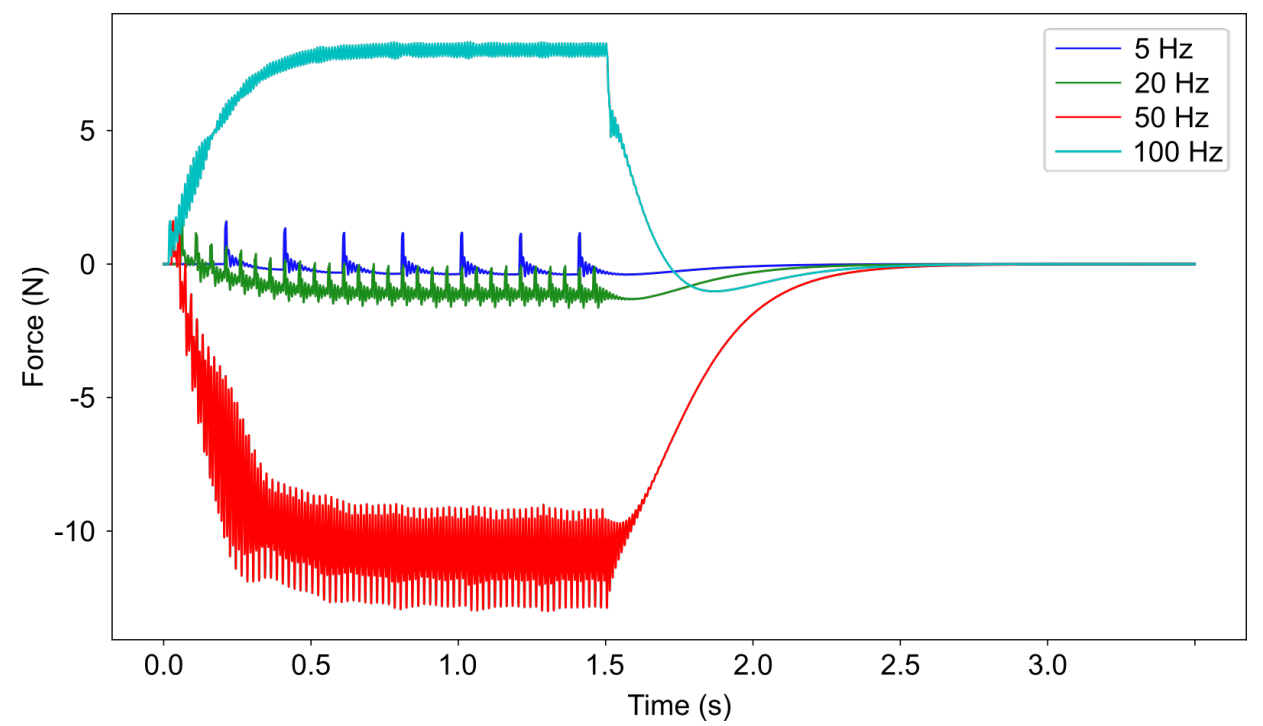

Figure 8: Differences of the force generated from muscles $M_{1}$ and $M_{2}$ according to the stimulation frequency.

\subsubsection{Motor unit scale}

Considering the model description, we have now access to the mechanical contribution developed by each MU. Thus, deeper investigation about the muscle mechanical behavior is feasible. In this section, we propose to dissect these simulated muscles at the MU scale in order to give some insights about the underlying mechanical phenomena.

On Fig. 9, the generated force according to the stimulation frequency and the MU type is presented. As one can see in this figure, the SMUs generate more force at $50 \mathrm{~Hz}$ than at 100 $\mathrm{Hz}$. Considering the slow contraction of such MUs, the tetanus contraction is obtained around $50 \mathrm{~Hz}$. The same effect can be observed on the FRMU type, where its optimal stimulation frequency is higher than the one for SMU. On the other hand, FIMU and FFMU have also this effect but with even a higher fusion frequency than SMU and FRMU. In this model, the optimal stimulation frequency is highly correlated to the calcium dynamic presented in 2.2.1. 

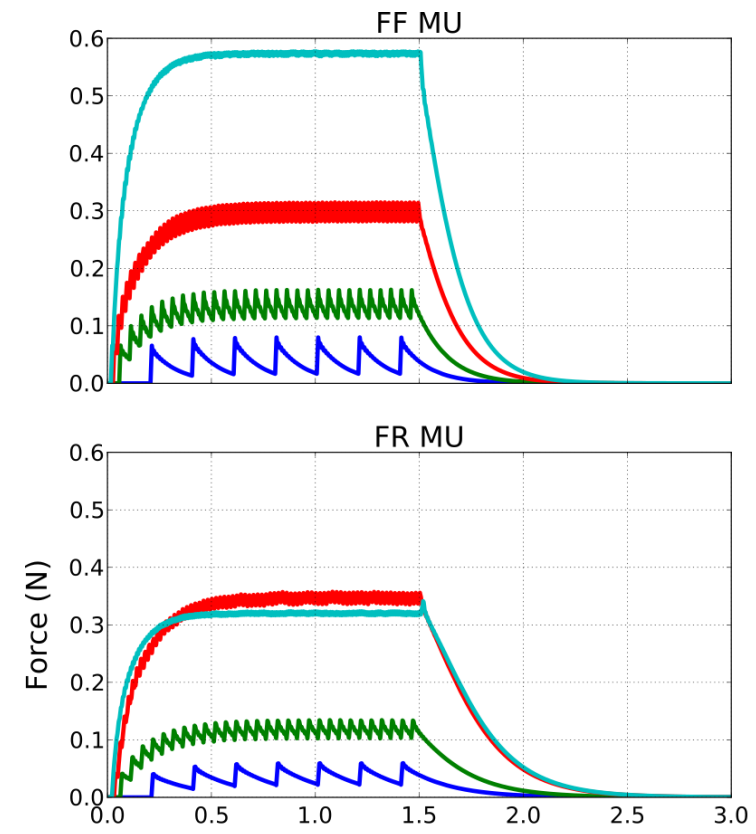
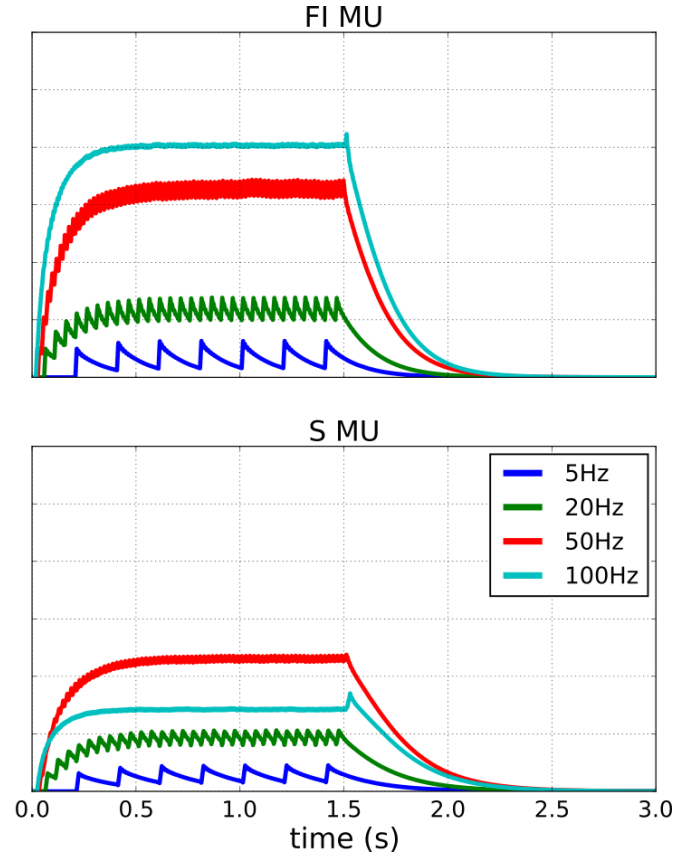

Figure 9: MU force response to the stimulation frequency according to its type.

\subsubsection{Comparison with the twitch model}

In this section, we will compare the force generated from a classical twitch model [26] using the same anatomy and stimulation frequency as depicted before (see Figs. 10 and 11). In [26], the twitch is a function of 6 parameters identified from experimental study.

$$
F(t)=p t^{m} e^{-k t}
$$

with, $p=F_{\max } e^{-k T_{c}\left(\ln \left(T_{c}\right)-1\right)}, m=k T_{c}$ and $k=\frac{\ln (2)}{-T_{c} \ln \left(\frac{T_{h r}}{T_{c}}\right)+T_{h r}-T_{c}} \cdot T_{c}$ represents the contraction time, the time from the start of $\mathrm{MU}$ force generation to the time where $\mathrm{MU}$ reaches its peak value $F_{\max }$. $T_{h r}$ describes the half time relaxation corresponding to the time from the start of MU force generation to the time where MU force decreases to half of its peak. Moreover, a time delay $T_{\text {lead }}$ representing the electromechanical delay is given to delay the response. Value of the parameters governing this model is presented in Table 4.

From this definition, the muscle force is computed as:

$$
F_{m}=\sum_{i=1}^{N} F_{i}(t) * \sum_{j=0}^{M} t_{k}
$$

where $F_{m}$ is the muscle force, $F_{i}$ is the twitch definition (see eq 27 ) of the $\mathrm{i}^{\text {th }} \mathrm{MU}, N$ is 
Table 4: Parameters used for the twitch model developped in [28]

\begin{tabular}{|c|c|c|c|c|}
\hline MU type & $T_{\text {lead }}(\mathrm{ms})$ & $T_{c}(\mathrm{~ms})$ & $T_{h r}(\mathrm{~ms})$ & $F_{\max }(\mathrm{N})$ \\
\hline $\mathrm{S}$ & 70 & 80 & 200 & 0.0382 \\
\hline $\mathrm{FR}$ & 60 & 70 & 175 & 0.0764 \\
\hline $\mathrm{FI}$ & 30 & 40 & 100 & 0.191 \\
\hline $\mathrm{FF}$ & 20 & 30 & 60 & 0.328 \\
\hline
\end{tabular}

the number of $\mathrm{MU}, t_{k}$ is the $\mathrm{k}^{t h}$ discharge time of the $\mathrm{MU}, M$ is the number of discharge and $*$ the convolution operator.

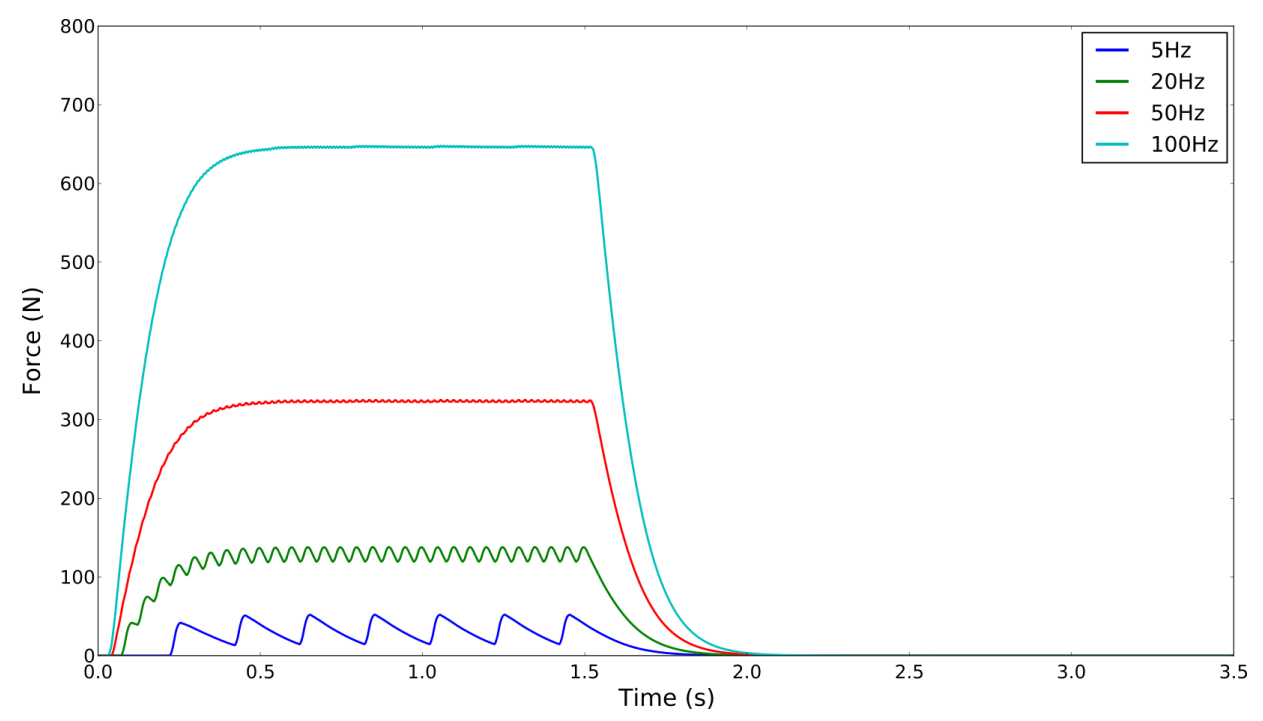

Figure 10: Fused and unfused tetani according to the stimulation frequency for muscle $\mathrm{M}_{2}$ with twitch model where all the muscle MU are recruited as in Fig. 7.

On Fig. 10, we can see a continuous increase in the generated force while increasing the stimulation frequency. Compared to Fig. 7, where we can observe an optimal frequency, the twitch model cannot describe this optimal frequency in this range of stimulation frequency. No saturation of tetanus can be visible with the twitch model. In fact, in the twitch model, the muscle force is computed from the sum of the convolution between the firing times of the MU and their corresponding twitch. Because of this convolution operator and the definition of the firing times of the MUs (Dirac impulse), it does not exist a generated force limit if 
$M \rightarrow \infty$. Thus, the increase of the stimulation frequency will define $\infty$ number of firing times.
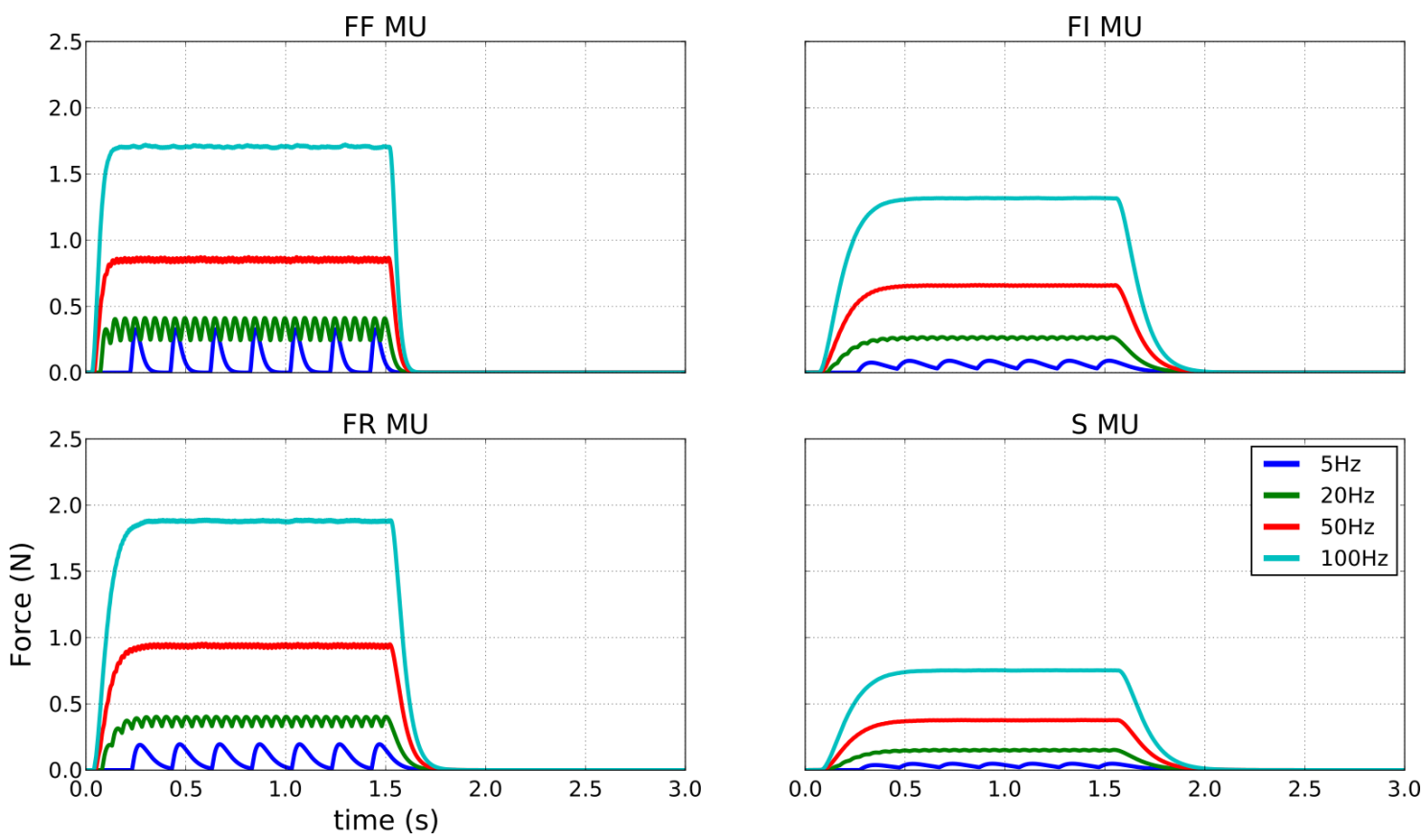

Figure 11: MU force response to the stimulation frequency according to its type using the twitch model.

At the MU scale, the same trend is observed on the generated force, which continuously increases with the stimulation frequency whatever the MU type (see Fig. 11). Compared to Fig. 9, the FRMU generates more force than FF or FIMUs that is not in agreement with the literature since the FFMUs are the MUs that produce the highest force. Thus, we can assess that the twitch model cannot transcribe an optimal stimulation frequency and is meaningless when high stimulation frequency $(>100 \mathrm{~Hz})$ is considered.

\subsection{Voluntary contraction simulation}

In the proposed model, the input parameter $\alpha$ describing the activation of the muscle is also adapted to MU voluntary recruitment. This formalism also allows us to simulate voluntary contraction as presented in Fig. 12.

Force profiles were generated using the same voluntary recruitment patterns defining force plateaus from 10 to $100 \%$ MVC. We are comparing the force profiles generated using the twitch model defined in [28] and the proposed mechanical model from the same recruitment 


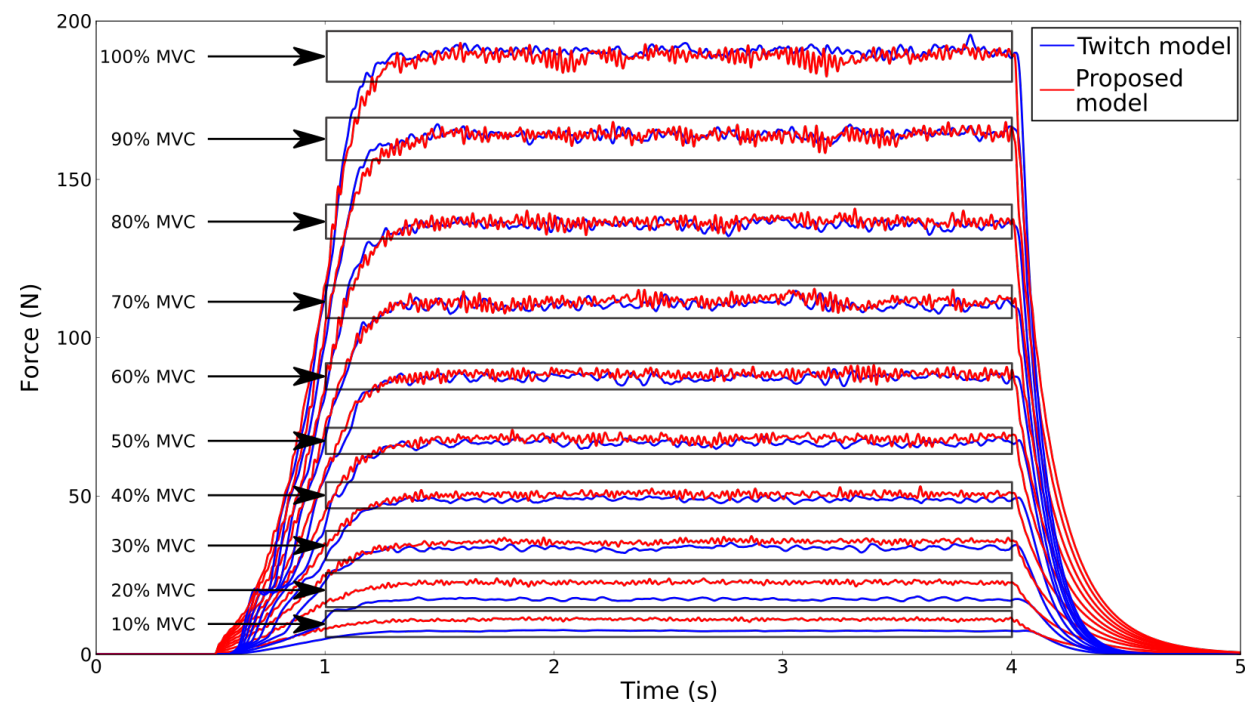

Figure 12: Force profiles for voluntary contractions from 10 to $100 \%$ MVC using the twitch model described above (blue) and the proposed muscle mechanical model (red).

pattern. The anatomy used is the $\mathrm{M}_{1}$ muscle one described in the previous section 3.1. Moreover, we notice that the parameters remain the same among the simulations for both models, only the recruitment pattern changes at each contraction.

As it can be observed in Fig. 12, the plateau remains stable along with the contraction with slight variability due to the MU recruitment rolling. The proposed model correctly represents the same force profiles generated from the validated twitch model [26]. In fact, the Normalized Root Mean Squared Error (NRMSE) is $5.51 \%$ between normalized force profiles for every contraction level. Only for the 10 and $20 \%$ MVC contractions, the proposed model simulates a higher force than the twitch model (at 10\% MVC NRMSE is $126 \%$ and $74 \%$ at $20 \% \mathrm{MVC}$ ). A comparison of the mean and the standard deviation (std) computed over the force plateau for both models is presented in Fig. 13a and 13b.

On Fig. 13a, we can observe almost the same mean computed over the force profiles plateau from both models (NRMSE is $1.26 \%$ ). Only the mean computed with the twitch model at 10 and $20 \%$ MVC is underestimated compared to the proposed model $(-48.52 \%$ and -29.98\%). Moreover, in Fig. 13b, is presented the standard deviation computed for both models. As we can see, more dispersion of the generated force is observed with the proposed model compared to the twitch model. Yet, the trend remains the same, the std increases 


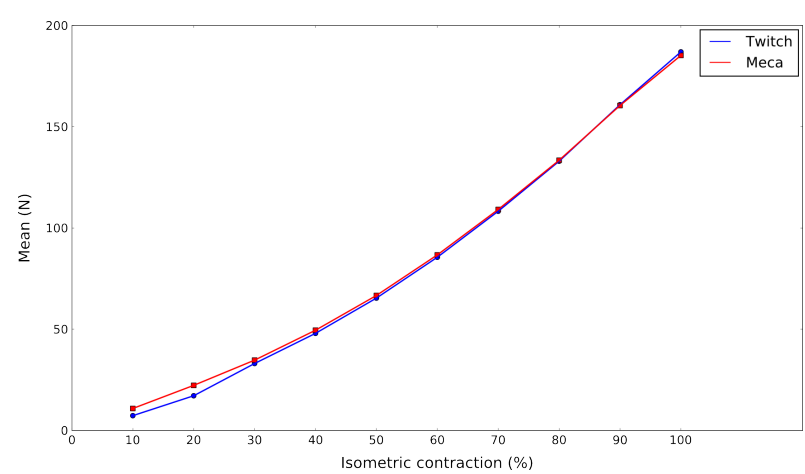

(a) Computed mean force for each contraction level according to the proposed model

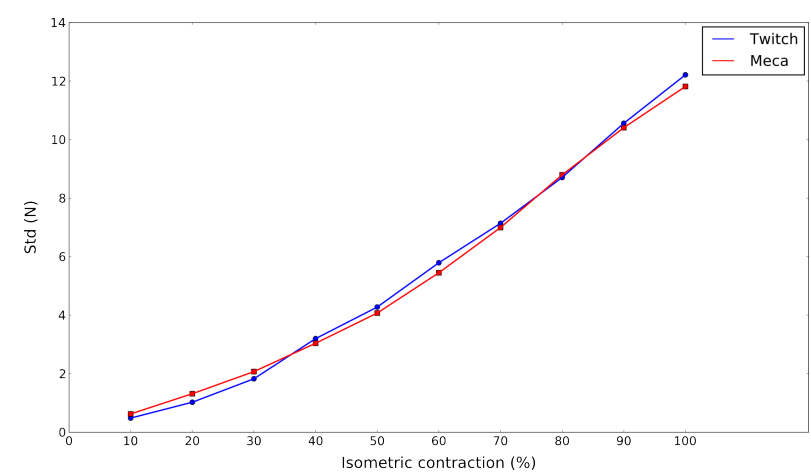

(b) Computed standard deviation (std) force for each contraction level according to the proposed model

Figure 13: Mean and std force computed from both models from 10 to $100 \%$ MVC.

according to the increase of the contraction level. Mean and std increasing according to the contraction level are also observed in experimental recordings [29, 30].

Otherwise, with the proposed model, we also have access to the muscle stiffness and deformation at each simulated instant of the contraction. Figures 14a and 14b present the computed mean for the stiffness and the deformation respectively.

According to Fig. 14a, the muscle stiffness continuously increases with the contraction level. The stiffness range value according to the contraction level is in agreement with experimental studies recording the muscle stiffness during contraction [31]. On Fig. 14b, one can see that the muscle continuously shortens according to the muscle activation level. At $100 \%$ MVC, the muscle length is around $2.4 \%$ shorter than its defined optimal length supposing less effectiveness of the force generation according to the defined force-length relationship (see eq (25)).

\subsection{Model validation}

Experimental data used in this study come from the study [4]. Particularly, these data were used to identify the parameters of a multiscale musculoskeletal model controlled by electrical stimulation. Details about the experimental setup and the identification process can be found in [4]. In this study, one force profile from isometric measurements recorded from the paraplegic subject implanted with an electrical stimulation device was used (see 


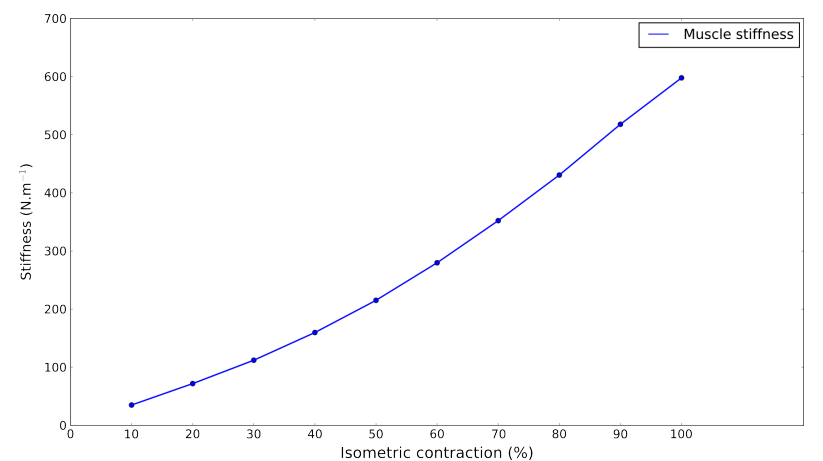

(a) Computed mean stiffness for each contraction level according to the proposed model

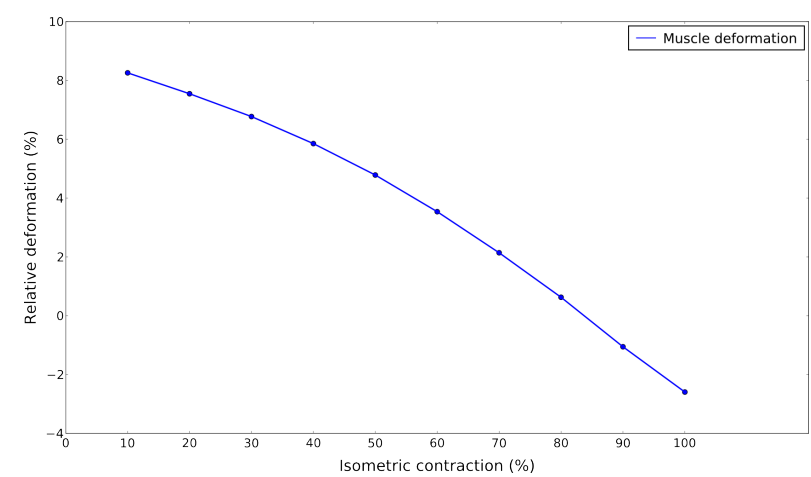

(b) Computed mean deformation for each contraction level according to the proposed model

Figure 14: Mean stiffness and deformation computed from the proposed model from 10 to $100 \%$ MVC.

[32] for the detailed clinical setup). During the stimulation, the knee joint was fixed at the position at which the torque was maximum. The quadriceps muscle was stimulated with 8 stimulation trains (duration $1.5 \mathrm{~s}$ ), separated by a $2 \mathrm{~s}$ rest time. The neural stimulation was performed using an implanted FES system [33] i.e. bipolar neural stimulation on the femoral nerve. Stimulation frequency was set at $20 \mathrm{~Hz}$ for the subject. Pulse widths were fixed during the stimulation, but the intensity increased between trains from $0.4 \mathrm{~mA}$ to maximum $\mathrm{i}_{\max }=$ $3.15 \mathrm{~mA}$ with a constant step $(\min .50 \mu \mathrm{A})$. These data are unique because neural implanted stimulation allows performing highly reproducible responses to stimulation and provide a full contraction of the targeted muscle without stimulating undesired muscle owing to the selection of fascicles during the initial surgery.

\subsubsection{Simulation of a quadriceps bulk}

The aim of this section is to validate the presented model according to the experimental recording. For this purpose, simulation parameters concerning the simulation are presented in Tables 1, 2 and 5. Some of these parameters were extracted from [4] while others were defined based on the literature $[34,35,36,37]$.

Considering that the stimulated muscle is the quadriceps, we decided in a first step to simulate a quadriceps bulk regrouping the four muscles composing the quadriceps as in [4]. For this purpose, we defined a muscle composed of 3250 MUs with the following distribution 
Table 5: Parameters used for the validation simulation

\begin{tabular}{|c|c|}
\hline Parameter & Value (unit) \\
\hline Maximal intensity $\left(i_{\max }\right)$ & $3.15(\mathrm{~mA})[4]$ \\
\hline Intensities $(i)$ & $0.4,0.8,1.2,1.6,2.0,2.4,2.8,3.15(\mathrm{~mA})[4]$ \\
\hline Ellicited recruitment parameters $\left(c_{1}, c_{2}, c_{3}\right)$ & $1.15,8.12,0.75[4]$ \\
\hline Optimal muscle length $\left(L_{c 0}\right)$ & $9.18(\mathrm{~cm})[4]$ \\
\hline Rest muscle length $\left(L_{c}^{0}\right)$ & $10.5(\mathrm{~cm})$ \\
\hline Velocity contribution $(a)$ & $1.0[4]$ \\
\hline Force-length parameter $(b)$ & $0.54[4]$ \\
\hline Tendon viscosity $(\lambda)$ & $19\left(\mathrm{~kg} . \mathrm{s}^{-1}\right)[10]$ \\
\hline Muscle weight $(m)$ & $1.6(\mathrm{~kg})[35]$ \\
\hline Maximal force $\left(F_{\max }\right)$ & $1000(\mathrm{~N})$ \\
\hline Maximal stiffness $\left(k_{m a x}\right)$ & $10000\left(\mathrm{~N} . \mathrm{m}^{-1}\right)[30]$ \\
\hline Tendon stiffness $\left(k_{s 1}\right)$ & $40000\left(\mathrm{~N} . \mathrm{m}^{-1}\right)[30]$ \\
\hline
\end{tabular}

40\% SMU, 13\% FRMU, 16\% FIMU and 31\% FFMU. This distribution is determined as a weighted average according to each muscle composition based on $[37,38]$ and the number of MUs. Here, we assumed that the rectus femoris (RF) muscle is composed of 750 MUs with an innervation ratio $\simeq 750$. This innervation ratio represents the average number of fiber innervated by the MUs. Thus, FF and FI MUs innervate more than 750 fibers and FR and S MUs less. The vastus intermedius (VI) has 500 MUs innervating around 750 fibers each. The vastus medialis (VM) has 1000 MUs innervating around 1000 fibers each. Finally, the vastus lateralis (VL) has 1000 MUs innervating around 1000 fibers each. In total, the defined muscle is composed of $\simeq 2,500,000$ muscle fibers.

Simulated and experimental force profiles were normalized according to their respective maximum. The first observation in Fig. 15 concerns the global dynamic of the force generation according to the stimulation intensity. Compared to experimental recording, the simulated force correctly describes the trend of experimental force. Only one force generation according to the stimulations (the $3^{\text {rd }}$ ) is underestimated while the $5^{\text {th }}$ stimulation is 


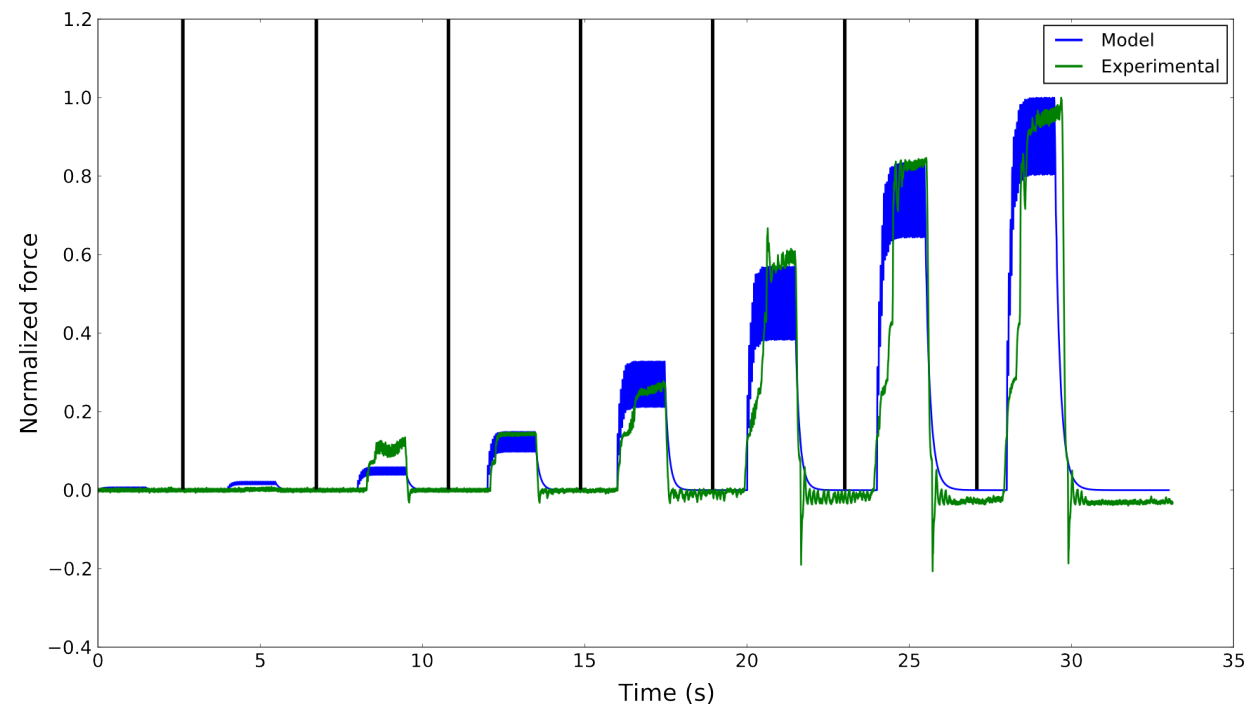

Figure 15: Normalized generated force in experimental conditions following the protocol presented above (data extracted from [4]) compares to simulated force from the proposed mechanical muscle model. Each stimulation train is separated by black lines.

overestimated. Moreover, we can observe the experimental recording a two steps increase of the force. After a few stimulations, the muscle force increases and stabilizes during a few hundreds of milliseconds and then continues to rise. This sidestepping rise is always the same between the stimulation whatever the intensity. To assess the effectiveness of the model, the Normalized Root Mean Square Error (NRMSE) was computed as follows:

$$
N R M S E=\frac{1}{(\max (\widehat{F})-\min (\widehat{F}))} \sqrt{\frac{\sum_{i=1}^{P}\left(\widehat{F}_{i}-F_{i}\right)^{2}}{P}}
$$

where, $P$ is the number of time sample of the force, $\hat{F}$ is the experimental force and $F$ is the simulated force. Using this equation, computed NRMSE indicates that the error between experimental and simulated force is $7.0 \%$. The major error comes from the almost perfectly synchronization of the muscle contraction since we only simulated one muscle representing the quadriceps. Moreover, the pennation angle influencing the force generation of each muscle is not considered and induce also possible error.

On Fig. 16, is exhibited the relationship between the generated force and the stimulation intensity. As one can see, the simulated forces closely fit the measured one, with a NRMSE of $4.6 \%$. This error is mainly due to the underestimation of the generated force of the 


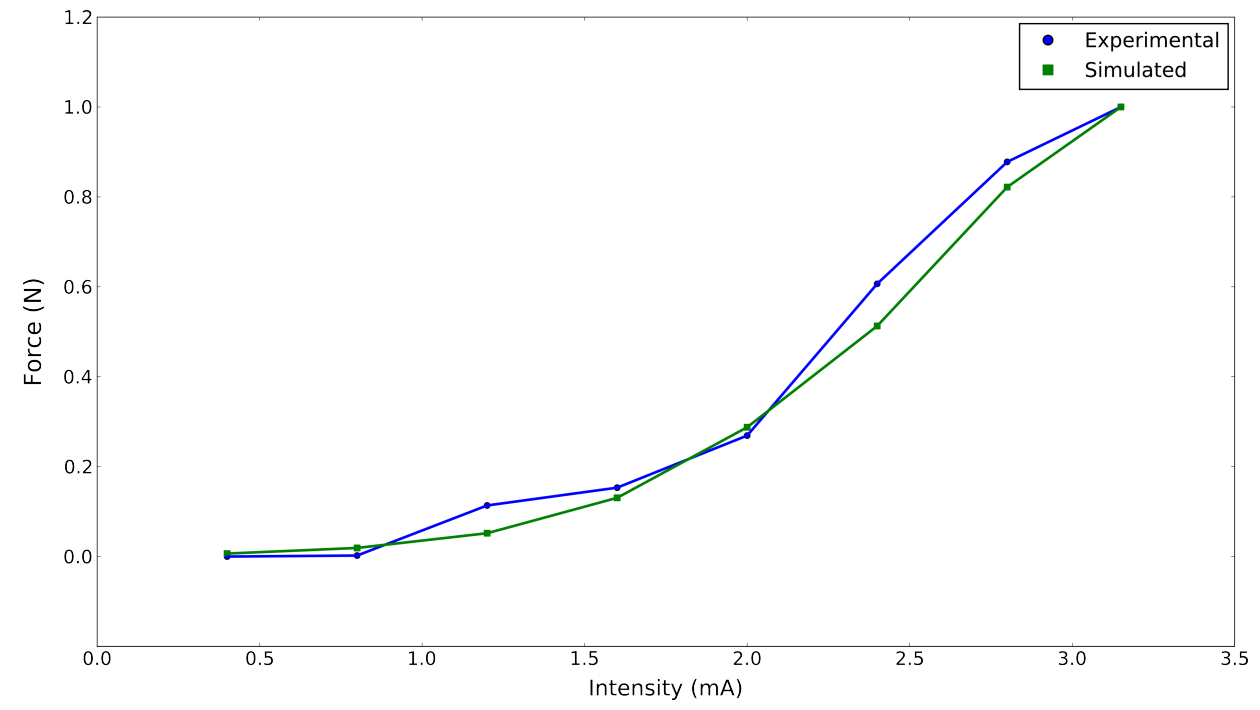

Figure 16: Mean of the normalized generated force in experimental conditions with the proposed mechanical muscle model according to the electrical stimulation intensity.

mechanical model compared to the experimental data. However, the relationship trend is preserved with a continuous increase of the force according to the stimulation intensity.

Finally, concerning the computation time of the model, this simulation lasts $57 \mathrm{~min}$ using a dedicated workstation $(2 \times 8$ cores Intel Xeon $2.40 \mathrm{Ghz}$ with hyperthreading, 128Gb Ram, Ubuntu 14.04 64bits) without parallel computation and using a unique processor. Naturally, this computing time can be drastically reduced using parallel computing paradigm for future applications.

\section{Discussion}

In this study, we proposed to model the mechanical behavior through the mechanical contractile properties of the skeletal muscle during isometric contraction. This model was described at the scale of the MUs, which are assumed to be placed as $N$ parallel contractile elements. In order to portray the MU mechanical behavior in a realistic manner, muscle MU typing and distribution is a crucial physiological and anatomical strategy for ensuring motor function diversity. This is often impacted in pathological conditions (sarcopenia, myopathies, etc.). Modeling the mechanical contribution of the muscle at the MU scale had previously been used in different studies $[13,26]$. Nevertheless, these models only computed the force 
contribution of each MU individually. In the work of Riener and al. [13], they described the calcium dynamics induced by the recruitment of the MU. However, some important relationships such as the force-length relationship were not considered in this work.

Determining the muscle force contribution is an association of several mechanisms and interactions. Most of these mechanisms are well described in $[5,6,7]$. However, in $[5,7]$ the muscle was described only as one contractile element where its activation did not have any physiological meaning. In [6], the muscle was described at the fiber scale and accurately characterized the underlying processes arising during muscle fiber contraction. But, using this model to define each fiber in the muscle is not feasible in terms of computation time for bio-reliable modeling used in clinical applications. Using these models tends to define the muscle activation during the contraction which is usually estimated from the corresponding recorded sEMG signal $[7,9,39,40,41]$. This estimation is reasonable since it is well known that the muscle activation is correlated to the sEMG signal amplitude but not necessarily in a linear manner [7, 29]. We also know that the sEMG signal can suffer from the autocancellation phenomenon [42] and the attenuation effect that leads to underestimated neural intent. Also, the electrical signal can record neighboring muscle electrical activity inducing crosstalk [43]. Another important issue is the spatial representativeness of a unique sEMG signal to estimate the muscle activation input. This problem has been recently partially solved using High-Density sEMG (HD-sEMG) technique [29, 44] where it has been demonstrated, by simulation, that using fusion technique on the HD-sEMG signals is more accurate to estimate the muscle activation. In the proposed model, this activation is estimated from the discharge times of each MU during contraction rather than using sEMG signal intensity. This major innovation opens the doors to possible studies linking neural drive strategies and bio-reliable production of muscle force. However, estimating the MU recruitment scheme from decomposing experimental sEMG activities is still a scientific challenge [45].

An important compromise between the Hill-type and Huxley-type models proposed in [8] using the distribution moment theory, allowed to upscale from the fiber scale to the muscle scale and thus, preserved the fiber accurate modeling to the muscle scale. This model gives promising results. There have been even some studies that upgraded this model as in the work of Makssoud and al. [10]. In [10], the authors also defined the input parameter $\alpha$ 
according to the functional electrical stimulation. Nevertheless, using the formalism proposed by Zahalak implies modeling simplification such as the muscle composition. In fact, applying the distribution moment theory proposed in [8] will consider that the muscle is composed of $N$ times the same muscle fiber.

In our model, we used the formalism proposed in [10] since this model was validated on human recordings. But, the major improvement is the description of the muscle as $N$ (number of MUs) contractile elements placed in parallel (see Fig. 6) where each contractile element is activated independently from the others. Actually, except for the activation, there is a strong dependence between the contractile element contraction and the global muscle contraction since muscle force-length and muscle force-velocity relationships are considered. The mechanical dynamic of this contractile element considered local variables as well as global variables at the muscle scale. Compared to [10], we specified the activation dynamic according to the MU type. We also decomposed the muscle as $N$ MUs placed in parallel with a specific mechanical contribution for each MU. In the anatomical model, we supposed a different amount of fiber according to the MU type. Thus, FFMUs have more fiber than SMUs in order to provide more contribution to the muscle contraction.

This is the first model, to our knowledge, that considers the mechanical relationships (force-length and force-velocity) and is able to simulate realistic mechanical voluntary contractions at the MU scale based on the discharge times of each MU composing the studied muscle. Moreover, compared to the twitch model, the proposed dynamic model is more complex and can simulate physiological behaviors that the twitch model cannot (see section 3.1). In fact, the twitch model cannot represent this fusion frequency compared to the proposed mechanical model (see section 3.1). It also does not represent the muscle stiffness and the muscle deformation which affect the muscle force dynamic where the proposed model does (see equation (23) and Fig. 14a). This model presented a continuous increase of the force generation for all the MUs. However, the proposed model exhibited a decrease in the force generation compared to $100 \mathrm{~Hz}$ of stimulation frequency for all MUs. Beyond the fusion frequency, we should observe an increase followed by a plateau of the force generation, not a decrease as seen only for SMUs. This decrease of force generation beyond the fusion fre- 
quency could be explained since above this fusion frequency the FFMUs will generate more force inducing more important muscle deformation. A confusion could thus appear with the contribution of the force-length relationship that applies separately for each MU type. However, the total force increases with the frequency following an already well-established statement.

Despite the promising results, we estimated that a comparison with experimental recordings during voluntary isometric contraction was not suitable yet. In fact, experimental recordings describe the global torque applied to a specific joint. Moreover, noises in the recorded signals and lack of training for force sustaining induces unsteady force plateaus (even for low contraction level) and thus, bias the comparison. Estimation of the recruitment instants of the active MUs is also hard and complex to perform [45]. Thus, comparison with experimental recordings can only be made on the statistics computed over the force profile and their trends according to force level.

Thus, the proposed model has been validated with experimental data under electrical stimulation context only [4]. In this context, the MU recruitment is not random but in a specific, even unknown, order. In fact, neural stimulation is very accurate and reproducible and thus, the input is an image of the neural drive, accurately controlled. Moreover, the muscle composition was roughly estimated and thus, the validation could provide an assessment of the ability of the model not only to estimate the global recruitment but also an estimation of the muscle composition. We chose this unique case, as we were able in previous studies $[4,10]$ to estimate all the biomechanical parameters with acceptable accuracy. It has to be noticed that no identification algorithm was used to identify the parameter values, since these values were extracted from the literature and experimental studies without an additional refining step. Most of the values of the parameters were found, but a major issue came during their collect: some parameter values were significantly different between studies representing the subjects' inter-variability and recording technique. On the other hand, some parameter values could not be found in the literature. Thus, for the simulations, we tried to define these values inside a physiological range, but a parameter identification algorithm is definitively needed to provide better results and the subject's specific parameter tuning. We thus could perform statistics, with more simulations, but we thought it is a first step to 
validate such a complex model in a well-controlled experiment. According to Fig. 15 and the small obtained NRMSE value, we can assess that the proposed model is quite accurate. Besides, the experimental setup measures the torque on the knee, not the direct output of the quadriceps muscle. The mechanical part linking the chair and the lower limber further induces slight modifications of the global dynamics due to small movements of the system: it smooths the torque and increases the time response. However, in isometric conditions, the final torque is almost a proportional image of the muscle force knowing that the neural stimulation used is targeting only the quadriceps. Thus, obtained results seem to be accurate and reliable.

The developed model does not consider the muscle fatigue. Yet, it is known that the electrical stimulation induced intense fatigue compared to voluntary contractions [46, 47]. This fatigue phenomenon is described at two different levels [48]:

- the neural fatigue representing the limitation of the nerves to generate sustained action potential;

- the muscular fatigue (or metabolic fatigue) representing the reduction of force generation by the muscle fibers.

Thus, the fatigue will induce a significant decrease in muscle force generation due to repeated muscular activation [49]. Considering the successive stimulations used in the experimental protocol (see section 3.3), the muscular fatigue phenomenon may occur during the recording as well as the potentiation effect at the beginning of the stimulation [50]. However, the data used were at a relatively low frequency and without extended stimulation periods so one can speculate that these phenomena should be relatively low but should be considered in further studies. The muscle fatigue is not considered in the proposed model but can be defined at the neural level as well as at the muscle level thanks to the decoupling of the neural input describing the MU firing times and the muscle definition. Moreover, the twitch potentiation phenomenon [51] is not described in the proposed model. This phenomenon is more pronounced for the fast twitch than in slow twitch fibers. It transcribes the augmentation of the twitch response following a brief muscle activation. Other physiological phenomena such as catch, sag [52] and cooperativity [53] are not yet considered in this model 
but can be included in further works. These phenomena are fiber-type specific and related to the calcium kinetics and will be added in further extension of the model. Furthermore, the proposed model should be used in macroscopic studies including agonist and antagonist muscles around a joint to evaluate, after model personalization, muscle synergies in several contexts like aging.

\section{Conclusion}

In this study, we proposed a mechanical model representing the muscle at the MU scale. Modeling the muscle at the MU scale allows us to have more information than the modeling at the muscle scale and also permits us to simulate a whole muscle compare to the fiber scale modeling. It also allows the simulation of mechanical behavior according to various muscle compositions.

This modeling at the MU scale can also provide the activation level of the muscle through the MUs firing times. This description provides us the possibility to define evoked contractions as well as voluntary contractions. To assess the validity of our model, we performed a validation comparison with experimental data recorded in a former study where we know exactly the recruitment induced by the electrical stimulation. The muscle force was simulated for a single muscle representing the quadriceps bulk composed of $3250 \mathrm{MUs}$ with the suspected fiber type distribution. The results exhibit reliable results compare to experimental recording with a NRMSE of 7.0\%. The relationship between the muscle force increase according to the intensity of the stimulation is respected.

Future works, using this model, are envisaged for more realistic study of the sEMG/force relationship as in [29]. In fact, this model can easily be used in parallel with an electrical model of the muscle contraction [20] where the input representing the MUs firing times and the muscle composition are the same. Using an electrical and the proposed mechanical muscle models can also assess the effectiveness of the muscle activation based on the sEMG signal $[39,40]$. Moreover, this proposed model can also be used for studying the musculoskeletal system of a specific joint. Currently, these studies used a Hill-type model describing the muscle contractile dynamics and estimate the muscle activation based on the corresponding sEMG signal $[54,55,56]$. 
Finally, a quasi-dynamic electro-mechanical model of the skeletal muscle during isometric contractions can be defined. Since the muscle deformation is determined with the corresponding MU recruitment pattern, we can use this deformation information in the electrical model to have a better representation of the muscle anatomy during contraction. Moreover, studies concerning the contribution of agonist and antagonist muscles can be performed using an identification algorithm and adapted MU recruitment pattern. A possible clinical application is related to the comprehension of sarcopenia, a degenerative loss of skeletal muscle mass quality and strength with aging, leading to frailty. Using the proposed model, after parameter identification and model personalization, one can simulate a sarcopenic muscle with both mechanical and electrical manifestations, using the coupling of the proposed model and a recent electrical model [20] and assess its impact on force production and motion genesis. Also, the proposed model will help the clinicians to better understand the relationship between neural drive and the force generation for a specific muscle architecture (fiber and MU typing and distribution). In fact, thanks to the model, it becomes possible to evaluate the effect of a modification of the MU recruitment scheme on the force and stiffness generation process specifically to a personalized muscle architecture. For this purpose, sensitivity analysis can be performed to study the impact of the muscle parameters. Since this electro-mechanical model is developed in a bio-reliable way (using parameters with a physiological meaning), sensitivity analysis can be used for the inverse problem to identify patient-specific parameters and possibly the firing discharge of the MUs during the generated contraction.

\section{References}

[1] D. Jones, J. Round, A. de Haan, Skeletal Muscle from Molecules to Movement, Churchill Livingstone, Edinburgh, 2004.

[2] P. E. Crago, P. H. Peckham, G. B. Thrope, Modulation of muscle force by recruitment during intramuscular stimulation, IEEE transactions on bio-medical engineering 27 (1980) 679-684.

[3] W. K. Durfee, K. E. MacLean, Methods for estimating isometric recruitment curves of 
electrically stimulated muscle, IEEE Transactions on Biomedical Engineering 36 (1989) 654-667.

[4] M. Benoussaad, P. Poignet, M. Hayashibe, C. Azevedo-Coste, C. Fattal, D. Guiraud, Experimental parameter identification of a multi-scale musculoskeletal model controlled by electrical stimulation: application to patients with spinal cord injury, Medical \& Biological Engineering \& Computing 51 (2013) 617-631.

[5] A. V. Hill, The Heat of Shortening and the Dynamic Constants of Muscle, Proceedings of the Royal Society of London B: Biological Sciences 126 (1938) 136-195.

[6] A. F. Huxley, Muscle structure and theories of contraction, Progress in Biophysics and Biophysical Chemistry 7 (1957) 255-318.

[7] F. E. Zajac, Muscle and tendon: properties, models, scaling, and application to biomechanics and motor control., Critical reviews in biomedical engineering 17 (1988) 359-411.

[8] G. I. Zahalak, A distribution-moment approximation for kinetic theories of muscular contraction, Mathematical Biosciences 55 (1981) 89-114.

[9] E. J. Cheng, I. E. Brown, G. E. Loeb, Virtual muscle: a computational approach to understanding the effects of muscle properties on motor control, Journal of Neuroscience Methods 101 (2000) 117-130.

[10] H. E. Makssoud, D. Guiraud, P. Poignet, M. Hayashibe, P.-B. Wieber, K. Yoshida, C. Azevedo-Coste, Multiscale modeling of skeletal muscle properties and experimental validations in isometric conditions, Biological Cybernetics 105 (2011) 121-138.

[11] G. A. Tsianos, C. Rustin, G. E. Loeb, Mammalian muscle model for predicting force and energetics during physiological behaviors, IEEE Transactions on Neural Systems and Rehabilitation Engineering 20 (2012) 117-133.

[12] J. C. Coggshall, G. A. Bekey, A stochastic model of skeletal muscle based on motor unit properties, Mathematical Biosciences 7 (1970) 405-419. 
[13] R. Riener, J. Quintern, A physiologically based model of muscle activation verified by electrical stimulation, Bioelectrochemistry and Bioenergetics 43 (1997) 257-264.

[14] L. M. Mendell, The size principle: a rule describing the recruitment of motoneurons, Journal of Neurophysiology 93 (2005) 3024-3026.

[15] A. J. Fuglevand, D. A. Winter, A. E. Patla, Models of recruitment and rate coding organization in motor-unit pools, Journal of Neurophysiology 70 (1993) 2470-2488.

[16] C. G. Kukulka, H. P. Clamann, Comparison of the recruitment and discharge properties of motor units in human brachial biceps and adductor pollicis during isometric contractions, Brain Research 219 (1981) 45-55.

[17] C. J. D. Luca, P. Contessa, Hierarchical control of motor units in voluntary contractions, Journal of Neurophysiology 107 (2012) 178-195.

[18] F. S. Ayachi, S. Boudaoud, C. Marque, Evaluation of muscle force classification using shape analysis of the sEMG probability density function: a simulation study, Medical \& Biological Engineering \& Computing 52 (2014) 673-684.

[19] D. R. McNeal, Analysis of a model for excitation of myelinated nerve, IEEE transactions on bio-medical engineering 23 (1976) 329-337.

[20] V. Carriou, S. Boudaoud, J. Laforet, F. S. Ayachi, Fast generation model of high density surface EMG signals in a cylindrical conductor volume, Computers in Biology and Medicine 74 (2016) 54-68.

[21] S. Gehlert, W. Bloch, F. Suhr, Ca2+-Dependent Regulations and Signaling in Skeletal Muscle: From Electro-Mechanical Coupling to Adaptation, International Journal of Molecular Sciences 16 (2015) 1066-1095.

[22] H. Hatze, A general myocybernetic control model of skeletal muscle, Biological Cybernetics 28 (1978) 143-157.

[23] A. Wexler, J. Ding, S. Binder-Macleod, A mathematical model that predicts skeletal muscle force, IEEE Transactions on Biomedical Engineering 44 (1997) 337-348. 
[24] S. M. Baylor, S. Hollingworth, Simulation of Ca2+ movements within the sarcomere of fast-twitch mouse fibers stimulated by action potentials, The Journal of General Physiology 130 (2007) 283-302.

[25] S. M. Baylor, S. Hollingworth, Sarcoplasmic reticulum calcium release compared in slowtwitch and fast-twitch fibres of mouse muscle, The Journal of Physiology 551 (2003) $125-138$.

[26] R. Raikova, J. Celichowski, M. Pogrzebna, H. Aladjov, P. Krutki, Modeling of summation of individual twitches into unfused tetanus for various types of rat motor units, Journal of Electromyography and Kinesiology 17 (2007) 121-130.

[27] B. Dreibati, C. Lavet, A. Pinti, G. Poumarat, Influence of electrical stimulation frequency on skeletal muscle force and fatigue, Annals of Physical and Rehabilitation Medicine 53 (2010) 266-277.

[28] R. T. Raikova, H. T. Aladjov, Hierarchical genetic algorithm versus static optimization - investigation of elbow flexion and extension movements, Journal of Biomechanics 35 (2002) 1123-1135.

[29] M. Al Harrach, V. Carriou, S. Boudaoud, J. Laforet, F. Marin, Analysis of the sEMG/Force Relationship using HD-sEMG Technique and Data Fusion: A simulation study, Computers in Biology and Medicine 83 (2017) 34-47.

[30] A. Lizu, Effect of the biceps Brachii tendon on elbow flexor force steadiness in men and women, Ph.D. thesis, University of British Columbia, 2015.

[31] E. Jarocka, J. Marusiak, M. Kumorek, A. Jaskólska, A. Jaskólski, Muscle stiffness at different force levels measured with two myotonometric devices, Physiological Measurement 33 (2012) 65.

[32] D. Guiraud, C. Azevedo Coste, M. Benoussaad, C. Fattal, Implanted functional electrical stimulation: case report of a paraplegic patient with complete SCI after 9 years, Journal of NeuroEngineering and Rehabilitation 11 (2014) 15. 
[33] D. Guiraud, T. Stieglitz, K. P. Koch, J.-L. Divoux, P. Rabischong, An implantable neuroprosthesis for standing and walking in paraplegia: 5-year patient follow-up, Journal of Neural Engineering 3 (2006) 268-275.

[34] R. Ando, A. Saito, Y. Umemura, H. Akima, Local architecture of the vastus intermedius is a better predictor of knee extension force than that of the other quadriceps femoris muscle heads, Clinical Physiology and Functional Imaging 35 (2015) 376-382.

[35] R. M. Erskine, D. A. Jones, C. N. Maganaris, H. Degens, In vivo specific tension of the human quadriceps femoris muscle, European Journal of Applied Physiology 106 (2009) 827.

[36] A. J. Blazevich, D. Cannavan, D. R. Coleman, S. Horne, Influence of concentric and eccentric resistance training on architectural adaptation in human quadriceps muscles, Journal of Applied Physiology 103 (2007) 1565-1575.

[37] M. A. Johnson, J. Polgar, D. Weightman, D. Appleton, Data on the distribution of fibre types in thirty-six human muscles: An autopsy study, Journal of the Neurological Sciences 18 (1973) 111-129.

[38] L. Acosta, R. R. Roy, Fiber-type composition of selected hindlimb muscles of a primate (cynomolgus monkey), The Anatomical Record 218 (1987) 136-141.

[39] T. S. Buchanan, D. G. Lloyd, K. Manal, T. F. Besier, Neuromusculoskeletal Modeling: Estimation of Muscle Forces and Joint Moments and Movements From Measurements of Neural Command, Journal of applied biomechanics 20 (2004) 367-395.

[40] M. Hayashibe, D. Guiraud, Voluntary EMG-to-force estimation with a multi-scale physiological muscle model, BioMedical Engineering OnLine 12 (2013) 86.

[41] A. A. Biewener, J. M. Wakeling, S. S. Lee, A. S. Arnold, Validation of Hill-type muscle models in relation to neuromuscular recruitment and force-velocity properties: predicting patterns of in vivo muscle force, Integrative and Comparative Biology 54 (2014) 1072-1083. 
[42] K. G. Keenan, D. Farina, K. S. Maluf, R. Merletti, R. M. Enoka, Influence of amplitude cancellation on the simulated surface electromyogram, Journal of Applied Physiology 98 (2005) 120-131.

[43] M. A. Harrach, B. Afsharipour, S. Boudaoud, V. Carriou, F. Marin, R. Merletti, Extraction of the Brachialis muscle activity using HD-sEMG technique and canonical correlation analysis, in: 2016 38th Annual International Conference of the IEEE Engineering in Medicine and Biology Society (EMBC), 2016, pp. 2378-2381.

[44] S. Boudaoud, S. Allouch, M. A. Harrach, F. Marin, On the benefits of using HDsEMG technique for estimating muscle force, Computer Methods in Biomechanics and Biomedical Engineering 18 (2015) 1890-1891.

[45] D. Farina, N. Jiang, H. Rehbaum, A. Holobar, B. Graimann, H. Dietl, O. C. Aszmann, The Extraction of Neural Information from the Surface EMG for the Control of UpperLimb Prostheses: Emerging Avenues and Challenges, IEEE Transactions on Neural Systems and Rehabilitation Engineering 22 (2014) 797-809.

[46] R. Riener, Model-based development of neuroprosthesis for paraplegic patients., Philosophical Transactions of the Royal Society B: Biological Sciences 354 (1999) 877-894.

[47] M. Papaiordanidou, D. Guiraud, A. Varray, Does central fatigue exist under lowfrequency stimulation of a low fatigue-resistant muscle?, European Journal of Applied Physiology 110 (2010) 815-823.

[48] R. M. Enoka, J. Duchateau, Muscle fatigue: what, why and how it influences muscle function, The Journal of Physiology 586 (2008) 11-23.

[49] T. Siatras, G. Poumarat, J. P. Boucher, J. C. Le Flohic, Normal and paralyzed muscle force and fatigability induced by electrical stimulation, Journal of Manipulative and Physiological Therapeutics 17 (1994) 321-328.

[50] M. Hayashibe, Q. Zhang, D. Guiraud, C. Fattal, Evoked emg-based torque prediction under muscle fatigue in implanted neural stimulation, Journal of Neural Engineering 8 (2011). 
[51] K. Sasaki, Y. Tomioka, N. Ishii, Activation of fast-twitch fibers assessed with twitch potentiation, Muscle \& Nerve 46 (2012) 218-227.

[52] J. Carp, P. Herchenroder, X. Chen, J. Wolpaw, Sag during unfused tetanic contractions in rat triceps surae motor units, Journal of neurophysiology 81 (1999) 2647-61.

[53] J. R. Moore, S. G. Campbell, W. Lehman, Structural determinants of muscle thin filament cooperativity, Archives of Biochemistry and Biophysics 594 (2016) 8-17.

[54] K. Manal, T. S. Buchanan, An Electromyogram-Driven Musculoskeletal Model of the Knee to Predict in Vivo Joint Contact Forces During Normal and Novel Gait Patterns, Journal of Biomechanical Engineering 135 (2013).

[55] A. Seth, M. Sherman, J. A. Reinbolt, S. L. Delp, OpenSim: a musculoskeletal modeling and simulation framework for in silico investigations and exchange, Procedia IUTAM 2 (2011) 212-232.

[56] J. Ambrósio, C. Quental, B. Pilarczyk, J. Folgado, J. Monteiro, Multibody biomechanical models of the upper limb, Procedia IUTAM 2 (2011) 4-17. 